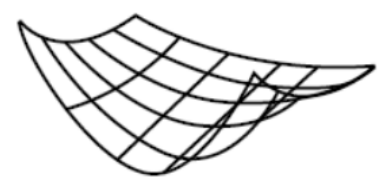

QUANTITATIVE FINANCE

RESEARCH CENTRE

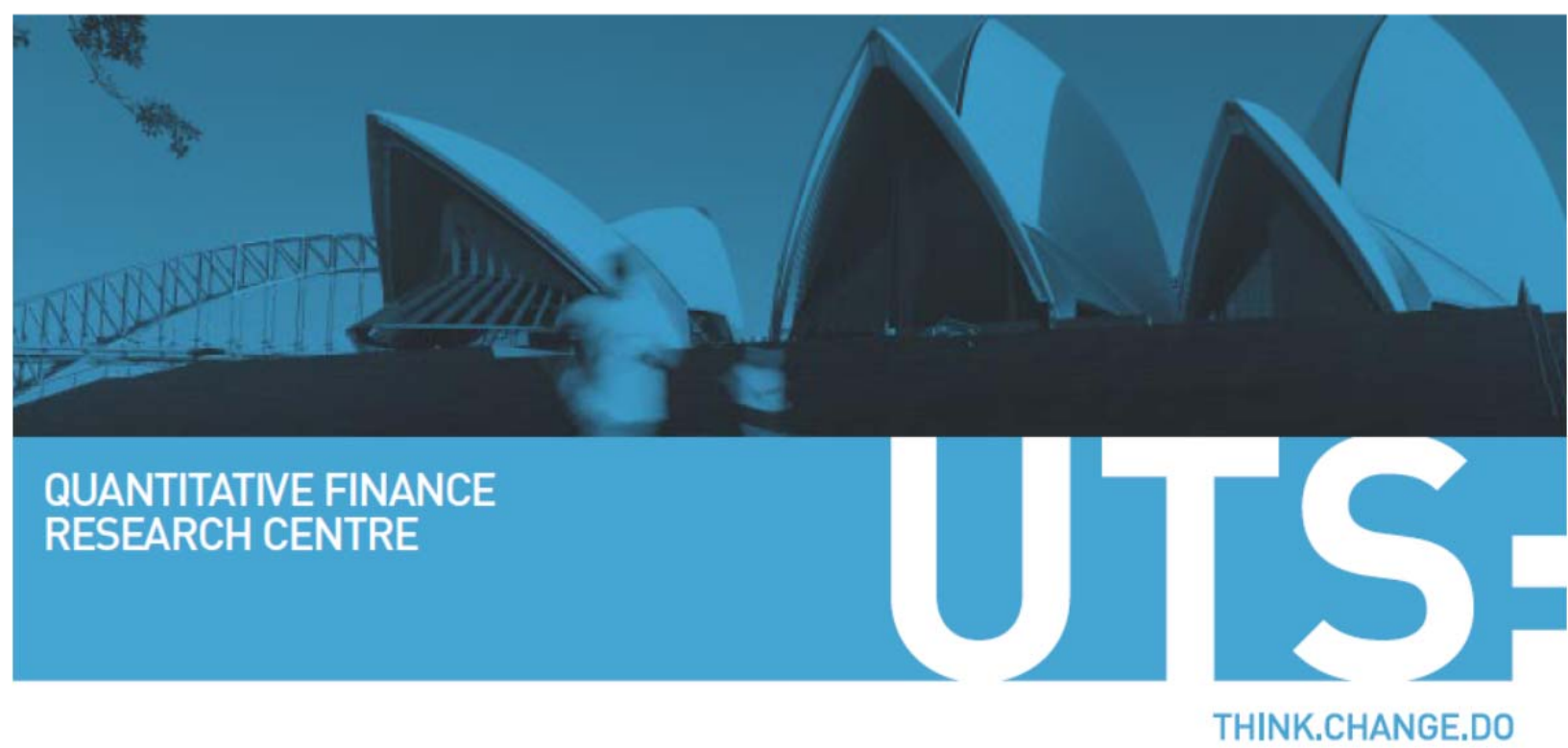

QuANTITATIVE FINANCE RESEARCH CENTRE

\title{
An Evolutionary CAPM Under \\ Heterogeneous Beliefs
}

Carl Chiarella, Roberto Dieci, Xue-Zhong He and Kai Li 


\title{
AN EVOLUTIONARY CAPM UNDER HETEROGENEOUS BELIEFS
}

\author{
CARL CHIARELLA*, ROBERTO DIECI**, XUE-ZHONG HE* AND KAI LI*
}

\author{
*Finance Discipline Group, UTS Business School \\ University of Technology, Sydney \\ PO Box 123, Broadway, NSW 2007, Australia \\ ** Department of Mathematics \\ University of Bologna \\ Piazza di Porta San Donato 5, I-40126 Bologna, Italy
}

Current version: September 25, 2012

Acknowledgement: We would like to thank Cars Hommes for a stimulating discussion in the early stages of this project. We are grateful to valuable comments from two anonymous referees. Dieci gratefully acknowledges a Visiting Professor Appointment at the Quantitative Finance Research Centre, UTS Business School, during which this work was finalised. Dieci also acknowledges support from MIUR under project PRIN 2009 "Local interactions and global dynamics in economics and finance: models and tools" and from EU COST within Action IS1104 "The EU in the new complex geography of economic systems: models, tools and policy evaluation". Financial support for Chiarella and He from the Australian Research Council (ARC) under Discovery Grant (DP110104487) is gratefully acknowledged.

Corresponding author: Xue-Zhong (Tony) He, Finance Discipline Group, UTS Business School, University of Technology, Sydney, email: tony.he1@uts.edu.au. Ph: (61 2) 9514 7726, Fax: (61 2) 95147722. 
ABSTRACT. Heterogeneity and evolutionary behaviour of investors are two of the most important characteristics of financial markets. This papers incorporates the adaptive behaviour of agents with heterogeneous beliefs and establishes an evolutionary capital asset pricing model (ECAPM) within the mean-variance framework. We show that the rational behaviour of agents switching to betterperforming trading strategies can cause large deviations of the market price from the fundamental value of one asset to spill over to other assets. Also, this spill-over effect is associated with high trading volumes and persistent volatility characterized by significantly decaying autocorrelations of, and positive correlation between, price volatility and trading volume.

Key words: Evolutionary CAPM, heterogeneous beliefs, market stability, spill-over effects, volatility, trading volume.

JEL Classification: D84, G12 


\section{INTRODUCTION}

Within the rational expectations and representative agent paradigm, the SharpeLintner-Mossin (Sharpe 1964, Lintner 1965 and Mossin 1966) Capital Asset Pricing Model (CAPM) is the most widely used tool to value returns on risky assets. However, there is considerable empirical evidence documenting cyclical behaviour of market characteristics, including risk premium, volatility, trading volume, price and dividend ratio, and in particular, market betas. The conditional CAPM was developed to provide a convenient way to incorporate time-varying beta and it exhibits empirical superiority in explaining the cross-section of returns and anomalies 11 There exists a large literature on time-varying beta models, but most of it is motivated by econometric estimation. It is often assumed that there are discrete changes in betas across subsamples but constant betas within subsamples 2 It has been shown that when betas vary over time, the standard OLS inference is misspecified and cannot be used to assess the fit of a conditional CAPM. In addition, most of the econometric models of time-varying beta lack any economic explanation and intuition.

Most theoretical models on conditional CAPM are based on the representative agent economy by assuming perfect rationality and homogeneous beliefs. However, empirical evidence along with unconvincing justification of the assumption of unbounded rationality and investor psychology, have led to the incorporation of both heterogeneity in beliefs and bounded rationality into asset pricing and financial market modelling (see the survey papers in the edited handbook of Hens and Schenk-Hoppé (2009) for developments in this literature). Heterogeneous beliefs and bounded rationality have become important parts of the asset pricing literature in recent years. Over the last two decades, there is a growing body of literature on heterogeneous agent models (HAMs) in economics and finance. HAMs consider

\footnotetext{
${ }^{1}$ See, for example, Engle (1982), Bollerslev (1986), Bollerslev et al (1988), Dybvig and Ross (1985), Hansen and Richard (1987), Hamilton (1989, 1990), Braun et al (1990), and Jagannathan and Wang (1996).

${ }^{2}$ See Campbell and Vuolteenaho (2004), Fama and French (2006), and Lewellen and Nagel (2006)). We point out that Ang and Chen (2007) treat betas as endogenous variables that vary slowly and continuously over time.
} 
financial markets as a nonlinear expectation-feedback system and reflect the interaction of heterogeneity, bounded rationality and adaptive behaviour of agents. Following the seminal work of Brock and Hommes (1997, 1998), various HAMs have been developed to show that, when agents increasingly switch to better performing strategies, this rational behaviour of agents can lead to instability of financial markets. This framework can also explain various types of market behaviour, such as the long-term swing of market prices from the fundamental prices, asset bubbles, market crashes, the stylized facts and various kinds of power law behaviour observed in financial markets 3

However, most of the HAMs analysed in the literature involve a financial market with only one risky asset and are not in the context of the CAPM. Recently, some attempts have been made to develop HAMs with many assets 4 Within a meanvariance framework, Chiarella et al (2010 and 2011) study a multi-asset CAPM through a consensus belief. In a dynamic setting, Chiarella et al (2012) demonstrate the stochastic behavior of time-varying betas and show that there can be inconsistency between ex-ante and ex-post estimates of asset betas when agents are heterogeneous and boundedly rational.

The aim of this paper is to extend the HAMs with one risky asset to an evolutionary CAPM within the framework of HAMs to examine the impact of adaptive behaviour of heterogeneous agents in a market with many risky assets. This paper is closely related to Chiarella et al (2012), but also differs from it in several respects. In

\footnotetext{
${ }^{3}$ We refer the reader to Hommes (2006), LeBaron (2006), Lux (2009), Chiarella et al (2009), Evstigneev, Hens and Schenk-Hoppé (2009) for surveys of the recent developments in this literature.

${ }^{4}$ We refer the reader to Chiarella et al (2005), Westerhoff and Dieci (2006), Chen and Huang (2008) and Marsili, Raffaelli and Ponsot (2009) for developments in multi-asset market dynamics in the literature of HAMs. In particular, Westerhoff (2004) considers a multi-asset model with fundamentalists who concentrate on only one market and chartists who invest in all markets; Dieci and Westerhoff $(2010 a, 2010 b)$ explore deterministic models to study two stock markets denominated in different currencies, which are linked via the related foreign exchange market; Chen and Huang (2008) develop a computational multi-asset artificial stock market to examine the relevance of risk preferences and forcasting accuracy to the survival of investors; and Marsili et al. (2009) introduce a generic model of a multi-asset financial market to show that correlation feedback can lead to market instability when trading volumes are high.
} 
Chiarella et al (2012), the heterogeneous beliefs are modelled at the return level and agents do not change their strategies. A spill-over effect of market instability from one asset to others due to behavioural change of agents is demonstrated through numerical simulations. In this paper, the heterogeneous beliefs are modelled at the price level and agents are allowed to change their strategies based on a fitness function similar to that used by Brock and Hommes $(1997,1998)$. The advantage of this setting is that we are able to examine the stability and spill-over effects analytically. We extend the single-period static model in Chiarella et al (2011) to a dynamic equilibrium asset pricing model. As in Chiarella et al (2012), we incorporate two types of investors, fundamentalists and trend followers, into the model. It is found that the instability of one asset, characterized by large fluctuations of market prices from the fundamental prices, can spill over to other assets when agents increasingly switch to better performing strategies. The spill-over effect is also associated with high trading volumes and persistent volatility, characterized by significantly positive and geometrically decaying autocorrelations in volume and volatility over long time horizons. Also the correlations between trading volume and volatility of risky assets are positive when asset payoffs are less correlated. These implications show that the evolutionary CAPM developed in this paper can provide insight into market characteristics related to trading volume and volatility. Also consistent with Chiarella et al (2012), we show that the commonly used rolling window estimates of time-varying betas may not be consistent with the ex-ante betas implied by the equilibrium model.

The paper is organized as follows. Section 2 sets up a dynamical equilibrium asset pricing model in the context of the CAPM to incorporate heterogeneous beliefs and adaptive behaviour of agents. Section 3 examines analytically the stability of the steady state equilibrium prices of the corresponding deterministic model. In Section 4 , we conduct a numerical analysis of the stochastic model to explore the spill-over effects, together with the relation between trading volumes and volatility, and the consistency of time-varying betas between ex-ante and rolling window estimates. Section 5 concludes. All proofs are given in the Appendix. 


\section{THE Model}

We consider an economy with $I$ agents, indexed by $i=1, \cdots, I$, who invest in portfolios consisting of a riskless asset with risk free rate $r_{f}$ and $N$ risky assets, indexed by $j=1, \cdots, N$ (with $N \geq 1$ ). Let $\mathbf{p}_{t}=\left(p_{1, t}, \cdots, p_{N, t}\right)^{\top}$ be the prices, $\mathbf{d}_{t}=\left(d_{1, t}, \cdots, d_{N, t}\right)^{\top}$ be the dividends and $\mathbf{x}_{t}:=\mathbf{p}_{t}+\mathbf{d}_{t}$ be the payoffs of the risky assets in period $t$ (from $t-1$ to $t$ ). Let $\mathbf{z}_{i, t}$ be the risky portfolio of agent $i$ (in terms of the number of shares of each risky asset), then the end-of-period portfolio wealth of agent $i$ is given by $W_{i, t+1}=\mathbf{z}_{i, t}^{\top}\left(\mathbf{x}_{t+1}-R_{f} \mathbf{p}_{t}\right)+R_{f} W_{i, t}$, where $R_{f}=1+r_{f}$.

2.1. Optimal Portfolio. Assume that agent $i$ has a constant absolute risk aversion (CARA) utility $u_{i}(x)=-e^{-\theta_{i} x}$, where $\theta_{i}$ is the CARA coefficient. Assuming that the wealth of agent $i$ is conditionally normally distributed, agent $i$ 's optimal investment portfolio is obtained by maximizing the certainty-equivalent utility of one-periodahead wealth 5

$$
U_{i, t}\left(W_{i, t+1}\right)=E_{i, t}\left(W_{i, t+1}\right)-\frac{\theta_{i}}{2} \operatorname{Var}_{i, t}\left(W_{i, t+1}\right) .
$$

Following Chiarella et al (2011), the optimal portfolio of agent $i$ is then given by

$$
\mathbf{z}_{i, t}=\theta_{i}^{-1} \boldsymbol{\Omega}_{i, t}^{-1}\left[E_{i, t}\left(\mathbf{x}_{t+1}\right)-R_{f} \mathbf{p}_{t}\right]
$$

where $E_{i, t}\left(\mathbf{x}_{t+1}\right)$ and $\boldsymbol{\Omega}_{i, t}=\left[\operatorname{Cov}_{i, t}\left(x_{j, t+1}, x_{k, t+1}\right)\right]_{N \times N}$ are respectively the conditional expectation and variance-covariance matrix of agent $i$ about the end-of-period payoffs of the risky assets, evaluated at time $t$.

2.2. Market Equilibrium. Assume that the $I$ investors can be grouped into $H$ agent-types, indexed by $h=1, \cdots, H$, where the agents within the same group are homogeneous in their beliefs as well as risk aversion. The risk aversion of agents of type $h$ is denoted by $\theta_{h}$. We also denote by $I_{h, t}$ the number of investors in group $h$ and by $n_{h, t}:=I_{h, t} / I$ the market fraction of agents of type $h$ in period $t$. Let $E_{h, t}\left(\mathbf{x}_{t+1}\right)$ and $\Omega_{h, t}=\left[\operatorname{Cov}_{h, t}\left(x_{j, t+1}, x_{k, t+1}\right)\right]_{N \times N}$ be respectively the conditional expectation and variance-covariance matrix of type- $h$ agents at time $t$. Let $\mathbf{s}=\left(s_{1}, \cdots, s_{N}\right)^{\top}$ be the $N$-dimensional vector of average risky asset supply per agent. A supply

\footnotetext{
${ }^{5}$ As is well known, the maximization of (2.1) is equivalent to maximizing the expected value of the above-defined CARA utility of wealth, $\max _{\mathbf{z}_{i, t}} E_{i, t}\left(u_{i}\left(W_{i, t+1}\right)\right)$, provided that $W_{i}$ is conditionally normally distributed in agent $i$ 's beliefs.
} 
shock to the market, denoted by a vector of random processes 6 is assumed to follow $\boldsymbol{\xi}_{t+1}=\boldsymbol{\xi}_{t}+\boldsymbol{\sigma}_{\kappa} \boldsymbol{\kappa}_{t+1}$, where $\boldsymbol{\kappa}_{t+1}$ is a standard normal i.i.d. random variable with $E\left(\boldsymbol{\kappa}_{t}\right)=\mathbf{0}$ and $\operatorname{Cov}\left(\boldsymbol{\kappa}_{t}\right)=\mathbf{I}$. Then the market clearing condition becomes

$$
\sum_{h=1}^{H} n_{h, t} \theta_{h}^{-1} \boldsymbol{\Omega}_{h, t}^{-1}\left[E_{h, t}\left(\mathbf{x}_{t+1}\right)-R_{f} \mathbf{p}_{t}\right]=\mathbf{s}+\boldsymbol{\xi}_{t} .
$$

2.3. Consensus Belief. We follow the construction in Chiarella et al (2011, 2012) to define an aggregate or consensus belief. Define the "average" risk aversion coefficient $\theta_{a, t}:=\left(\sum_{h=1}^{H} n_{h, t} \theta_{h}^{-1}\right)^{-1}$, which is a market population fraction weighted harmonic mean of the risk aversions of different types of heterogeneous agents. Specifically, if all agents have the same risk aversion coefficient $\theta_{h}=\theta$, then the "average" risk aversion coefficient $\left.\theta_{a, t}=\theta\right]$ Then the aggregate beliefs at time $t$ about variances/covariances and expected payoffs over the time interval $(t, t+1)$ are specified, respectively, as

$$
\begin{gathered}
\boldsymbol{\Omega}_{a, t}=\theta_{a, t}^{-1}\left(\sum_{h=1}^{H} n_{h, t} \theta_{h}^{-1} \Omega_{h, t}^{-1}\right)^{-1} \\
E_{a, t}\left(\mathbf{x}_{t+1}\right)=\theta_{a, t} \boldsymbol{\Omega}_{a, t} \sum_{h=1}^{H} n_{h, t} \theta_{h}^{-1} \boldsymbol{\Omega}_{h, t}^{-1} E_{h, t}\left(\mathbf{x}_{t+1}\right) .
\end{gathered}
$$

The dividend process $\mathbf{d}_{t}$ is assumed to follow a martingale process

$$
\mathbf{d}_{t+1}=\mathbf{d}_{t}+\boldsymbol{\sigma}_{\zeta} \boldsymbol{\zeta}_{t+1}
$$

where $\boldsymbol{\zeta}_{t+1}$ is a standard normal i.i.d. random variable with $E\left(\boldsymbol{\zeta}_{t}\right)=\mathbf{0}$ and $\operatorname{Cov}\left(\boldsymbol{\zeta}_{t}\right)=$ $\mathbf{I}$, independent of $\boldsymbol{\kappa}_{t+1}$. Moreover, agents are assumed to have homogeneous and correct conditional beliefs about the dividends (the unconditional expectation of which is assumed to be constant, $\left.E\left(\mathbf{d}_{t}\right)=\overline{\mathbf{d}}\right)$. Following Chiarella et al $(2011,2012)$, the market equilibrium prices (2.3) can therefore be rewritten as if they were determined by a homogeneous agent endowed with average risk aversion $\theta_{a, t}$ and the consensus beliefs $\left\{E_{a, t}, \Omega_{a, t}\right\}$, namely

$$
\mathbf{p}_{t}=\frac{1}{R_{f}}\left[E_{a, t}\left(\mathbf{p}_{t+1}\right)+\mathbf{d}_{t}-\theta_{a, t} \boldsymbol{\Omega}_{a, t}\left(\mathbf{s}+\boldsymbol{\xi}_{t}\right)\right] .
$$

\footnotetext{
${ }^{6} \sigma_{\kappa}$ is not necessarily a diagonal matrix, that is, the supply noise processes of the $N$ assets can be correlated. The same also holds for $\boldsymbol{\sigma}_{\zeta}$ in the dividend processes (2.5).

${ }^{7}$ This is the case that we use for the numerical analysis in Section 4.
} 
Note that at time $t$ the dividends $\mathbf{d}_{t}$ are realized and agents formulate their beliefs about the next period payoff $\mathbf{x}_{t+1}=\mathbf{p}_{t+1}+\mathbf{d}_{t+1}$, based on realized prices up to time $t-1$ and the dividends $\mathbf{d}_{t}$.

2.4. Fitness. Following the discrete choice model (discussed for instance by Brock and Hommes $(1997,1998)$, the market fractions $n_{h, t}$ of agents of type $h$ are determined by their fitness $v_{h, t-1}$, where the subscript $t-1$ indicates that fitness depends only on past observed prices and dividends. The fraction of agents using strategy of type $h$ is thus driven by "experience" through reinforcement learning. That is, given the fitness $v_{h, t-1}$, the fraction of agents using strategy type of $h$ is determined by the discrete choice model,

$$
n_{h, t}=\frac{e^{\eta v_{h, t-1}}}{Z_{t}}, \quad Z_{t}=\sum_{h} e^{\eta v_{h, t-1}}
$$

where $\eta>0$ is the switching intensity of choice parameter measuring how sensitive agents are to selecting a better preforming strategy 8 If $\eta=0$, then agents are insensitive to past performance and pick a strategy at random with equal probability. In the other extreme case $\eta \rightarrow \infty$, all agents choose the forecast that performed best in the last period. An increase in the intensity of choice $\eta$ can therefore represent an increase in the degree of rationality with respect to the evolutionary selection of strategies.

Given the utility maximization problem (2.1) of agents, we use a fitness measure that generalizes the 'risk-adjusted profit' introduced in Hommes (2001) (see Hommes and Wagener (2009) for a discussion about different choice of fitness functions and the relation), namely we set

$$
v_{h, t}=\pi_{h, t}-\pi_{h, t}^{B}-C_{h},
$$

where $C_{h} \geq 0$ measures the cost of the strategy,

$$
\pi_{h, t}:=\mathbf{z}_{h, t-1}^{\top}\left(\mathbf{p}_{t}+\mathbf{d}_{t}-R_{f} \mathbf{p}_{t-1}\right)-\frac{\theta_{h}}{2} \mathbf{z}_{h, t-1}^{\top} \boldsymbol{\Omega}_{h, t-1} \mathbf{z}_{h, t-1}
$$

and

$$
\pi_{h, t}^{B}:=\left(\frac{\theta_{a, t-1}}{\theta_{h}} \mathbf{s}\right)^{\top}\left(\mathbf{p}_{t}+\mathbf{d}_{t}-R_{f} \mathbf{p}_{t-1}\right)-\frac{\theta_{h}}{2}\left(\frac{\theta_{a, t-1}}{\theta_{h}} \mathbf{s}\right)^{\top} \Omega_{h, t-1}\left(\frac{\theta_{a, t-1}}{\theta_{h}} \mathbf{s}\right) .
$$

\footnotetext{
${ }^{8}$ In fact, $\eta$ is inversely related to the variance of the noise in the observation of random utility.
} 
Note that (2.9) can be naturally interpreted as the risk-adjusted profit of type $h$ agent. It represents the realized profit adjusted by the subjective risk undertaken by investor $h$, which is consistent with investors' utility-maximizing portfolio choices. Expression (2.10) can be interpreted as the (risk-adjusted) profit on portfolio $\mathbf{z}_{h, t-1}^{B}:=\frac{\theta_{a, t-1}}{\theta_{h}} \mathbf{s}$, which represents a 'benchmark' portfolio for agents of type $h$ at time $t-1$. The portfolio $\mathbf{z}_{h, t-1}^{B}$ is proportional to the market portfolio. The proportionality coefficient $\frac{\theta_{a, t-1}}{\theta_{h}}$ takes into account the fact that the shares of the market portfolio of agents are positively correlated to their risk tolerance $\left(1 / \theta_{h}\right)$. In the case that all agents have the same risk aversion ( so that $\theta_{a, t-1}=\theta_{h}$ ), they all take the market portfolio. Put differently, the performance measure (2.8) views strategy $h$ as a successful strategy only to the extent it outperforms its market benchmark in terms of risk-adjusted profitability. More precisely, portfolio $z_{h, t}^{B}$ represents the portfolio that agents of type $h$ would select at time $t$ if all agents had identical beliefs (whichever they are) about the first and second moment of $\mathbf{x}_{t+1}$. In this case agents would (possibly) differ only in terms of their risk aversion and $z_{h, t}=z_{h, t}^{B}$ for all $h$. According to the fitness measure $v_{h, t}:=\pi_{h, t}-\pi_{h, t}^{B}-C_{h}$, their portfolios would thus have identical performance (apart from the costs). In other words, the selected fitness measure $v_{h, t}$ is not affected by mere differences in risk aversion and accounts only of the profitability generated by the competing investment rules. Note that, in the case of zero supply of outside shares, the market clearing equation (2.6) leads to $E_{a, t}\left(\mathbf{x}_{t+1}\right) / R_{f}=\mathbf{p}_{t}$. This is the case considered in Hommes (2001) for a single-risky-asset model. The market thus behaves as if it were 'risk-neutral' at the aggregate leve 9 in this particular case and the performance measure reduces to the risk-adjusted profit considered in Hommes (2001).

2.5. Fundamentalists. Now we propose a model with classical heterogeneous agenttypes and consider two types of agents, fundamentalists and trend followers, or chartists, with $h=f$ and $h=c$, respectively. Following He and Li (2007), the fundamentalists realize the existence of non-fundamental traders, such as trend followers to be introduced in the following discussion. The fundamentalists believe that the stock price may be driven away from the fundamental value in the short-run,

\footnotetext{
${ }^{9}$ Of course, risk aversion does affect decisions at the agent-type level.
} 
but it will eventually converge to the expected fundamental value in the long-run. Hence the conditional mean of the fundamental traders is assumed to follow

$$
E_{f, t}\left(\mathbf{p}_{t+1}\right)=\mathbf{p}_{t-1}+\boldsymbol{\alpha}\left(E_{f, t}\left(\mathbf{p}_{t+1}^{*}\right)-\mathbf{p}_{t-1}\right)
$$

where $\mathbf{p}_{t}^{*}=\left(p_{1, t}^{*}, \cdots, p_{N, t}^{*}\right)$ is the vector of fundamental prices and the parameter $\boldsymbol{\alpha}=\operatorname{diag}\left[\alpha_{1}, \cdots, \alpha_{N}\right]$ with $\alpha_{j} \in[0,1]$ represents the speed of price adjustment of the fundamentalists toward their expected fundamental value or it reflects how confident they are in the fundamental value. The parameter $\alpha_{j}$ can be different for different risky assets. In particular, for $\alpha_{j}=1$, the fundamental traders are fully confident about the fundamental value of risky asset $j$ and adjust their expected price in the next period instantaneously to the expected fundamental value. For $\alpha_{j}=0$, the fundamentalists become naive traders of asset $j$. We also assume that the fundamentalists have constant beliefs about the covariance matrix of the payoffs so that $\boldsymbol{\Omega}_{f, t}=\boldsymbol{\Omega}_{0}:=\left(\sigma_{j k}\right)_{N \times N}$.

2.6. Fundamental Prices. To define the fundamental price $\mathbf{p}_{t}^{*}$, we consider a 'standard CAPM' with homogeneous beliefs where all agents have correct beliefs about the fundamental prices and are fully confident about their expected fundamental values (that is $\boldsymbol{\alpha}=\operatorname{diag}[1, \cdots, 1]$ ). We also assume that their average risk aversion coefficient is constant over time, $\theta_{a, t}=\bar{\theta}$, and so are their common secondmoment beliefs, $\Omega_{0} 10$ Correspondingly we define the fundamental price as

$$
\mathbf{p}_{t}^{*}=\frac{1}{r_{f}}\left(\mathbf{d}_{t}-\bar{\theta} \mathbf{\Omega}_{0}\left(\mathbf{s}+\boldsymbol{\xi}_{t}\right)\right),
$$

which is a martingale process under the assumptions about the exogenous dividend and market noise processes, so that

$$
\mathbf{p}_{t+1}^{*}=\mathbf{p}_{t}^{*}+\boldsymbol{\epsilon}_{t+1}, \quad \boldsymbol{\epsilon}_{t+1}=\frac{1}{r_{f}}\left(\boldsymbol{\sigma}_{\zeta} \boldsymbol{\zeta}_{t+1}-\bar{\theta} \boldsymbol{\Omega}_{0} \boldsymbol{\sigma}_{\kappa} \boldsymbol{\kappa}_{t+1}\right) \sim \text { Normal i.i.d. }
$$

In this case, it follows from Eq. (2.6) that the equilibrium price is given by $\mathbf{p}_{t}=\mathbf{p}_{t}^{*}$. Thus we can treat the benchmark CAPM case as the 'steady state' of the dynamics of the heterogeneous beliefs model.

\footnotetext{
${ }^{10}$ Without switching, the average risk aversion $\bar{\theta}$ is constant and corresponds to the harmonic mean of the risk aversion coefficients of all agents. In our simulations, we will set $\bar{\theta}=\theta_{a}^{*}$, the average risk aversion coefficient at the steady state solution of the model.
} 
2.7. Trend Followers. Unlike the fundamental traders, trend followers are technical traders who believe the future price change can be predicted from various patterns or trends generated from the historical prices. They are assumed to extrapolate the latest observed price change over a long-run sample mean price and to adjust their variance estimate accordingly. More precisely, their conditional mean and covariance matrices are assumed to satisfy

$$
E_{c, t}\left(\mathbf{p}_{t+1}\right)=\mathbf{p}_{t-1}+\gamma\left(\mathbf{p}_{t-1}-\mathbf{u}_{t-1}\right), \quad \mathbf{\Omega}_{c, t}=\Omega_{0}+\lambda \mathbf{V}_{t-1},
$$

where $\mathbf{u}_{t-1}$ and $\mathbf{V}_{t-1}$ are sample means and covariance matrices of past market prices $\mathbf{p}_{t-1}, \mathbf{p}_{t-2}, \cdots$, the constant vector $\gamma=\operatorname{diag}\left[\gamma_{1}, \cdots, \gamma_{N}\right]>0$ reflects the trend following strategy, and $\gamma_{j}$ measures the extrapolation rate and high (low) values of $\gamma_{j}$ correspond to strong (weak) extrapolation by trend followers, and $\lambda$ measures the sensitivity of the second-moment estimate to the sample variance. This specification of the trend followers captures the extrapolative behavior of the trend followers, who expect price changes to occur in the same direction as the price trend observed over a past time window. Assume that $\mathbf{u}_{t-1}$ and $\mathbf{V}_{t-1}$ are computed recursively as

$$
\begin{aligned}
\mathbf{u}_{t-1} & =\delta \mathbf{u}_{t-2}+(1-\delta) \mathbf{p}_{t-1}, \\
\mathbf{V}_{t-1} & =\delta \mathbf{V}_{t-2}+\delta(1-\delta)\left(\mathbf{p}_{t-1}-\mathbf{u}_{t-2}\right)\left(\mathbf{p}_{t-1}-\mathbf{u}_{t-2}\right)^{\top}
\end{aligned}
$$

Effectively, the sample mean and variance-covariance matrix are calculated based on the all historical prices $\mathbf{p}_{t-1}, \mathbf{p}_{t-2}, \cdots$, spreading back to $-\infty$ with geometric decaying probability weights $(1-\delta)\left\{1, \delta, \delta^{2}, \cdots\right\}$. Therefore, as $\delta$ decreases, the weights on the latest prices increase but decay geometrically at a common rate of $\delta$. For $\boldsymbol{\gamma}>\mathbf{0}$ and large $\delta$, momentum traders calculate the trend based on a long time horizon. In particular, when $\delta=0, E_{c, t}\left(\mathbf{p}_{t+1}\right)=\mathbf{p}_{t-1}$ and $\mathbf{V}_{t-1}=\mathbf{0}$, implying naive behaviour by the trend followers. However, when $\delta=1, \mathbf{u}_{t-1}=\mathbf{u}_{0}$ and $\mathbf{V}_{t-1}=\mathbf{V}_{0}$, and therefore $E_{c, t}\left(\mathbf{p}_{t+1}\right)=\mathbf{p}_{t-1}+\gamma\left(\mathbf{p}_{t-1}-\mathbf{u}_{0}\right)$, so that trend followers are momentum traders.

2.8. The Complete Dynamic Model. Based on the analysis above, the optimal demands of the fundamentalists and trend followers are given, respectively, by

$$
\mathbf{z}_{f, t}=\theta_{f}^{-1} \Omega_{o}^{-1}\left[\mathbf{p}_{t-1}+\mathbf{d}_{t}+\boldsymbol{\alpha}\left(\mathbf{p}_{t}^{*}-\mathbf{p}_{t-1}\right)-R_{f} \mathbf{p}_{t}\right]
$$


and

$$
\mathbf{z}_{c, t}=\theta_{c}^{-1}\left[\Omega_{0}+\lambda \mathbf{V}_{t-1}\right]^{-1}\left[\mathbf{p}_{t-1}+\mathbf{d}_{t}+\gamma\left(\mathbf{p}_{t-1}-\mathbf{u}_{t-1}\right)-R_{f} \mathbf{p}_{t}\right]
$$

Finally, the general dynamic model (2.6) reduces to the random nonlinear dynamical system

$$
\left\{\begin{aligned}
\mathbf{p}_{t}= & \frac{\theta_{a, t}}{R_{f}} \boldsymbol{\Omega}_{a, t}\left[\frac{n_{f, t}}{\theta_{f}} \boldsymbol{\Omega}_{0}^{-1}\left(\mathbf{p}_{t-1}+\boldsymbol{\alpha}\left(\mathbf{p}_{t}^{*}-\mathbf{p}_{t-1}\right)\right)\right. \\
& \left.+\frac{n_{c, t}}{\theta_{c}}\left(\boldsymbol{\Omega}_{0}+\lambda \mathbf{V}_{t-1}\right)^{-1}\left(\mathbf{p}_{t-1}+\gamma\left(\mathbf{p}_{t-1}-\mathbf{u}_{t-1}\right)\right)-\mathbf{s}-\boldsymbol{\xi}_{t}\right]+\frac{1}{R_{f}} \mathbf{d}_{t}, \\
\mathbf{p}_{t}^{*}= & \frac{1}{r_{f}}\left(\mathbf{d}_{t}-\theta_{a}^{*} \boldsymbol{\Omega}_{0}\left(\mathbf{s}+\boldsymbol{\xi}_{t}\right)\right), \\
\mathbf{u}_{t}= & \delta \mathbf{u}_{t-1}+(1-\delta) \mathbf{p}_{t}, \\
\mathbf{V}_{t}= & \delta \mathbf{V}_{t-1}+\delta(1-\delta)\left(\mathbf{p}_{t}-\mathbf{u}_{t-1}\right)\left(\mathbf{p}_{t}-\mathbf{u}_{t-1}\right)^{\top} \\
n_{f, t}= & \frac{1}{1+e^{-\eta v_{\Delta, t-1}}} \\
\boldsymbol{\xi}_{t}= & \boldsymbol{\xi}_{t-1}+\boldsymbol{\sigma}_{\kappa} \boldsymbol{\kappa}_{t}, \\
\mathbf{d}_{t}= & \mathbf{d}_{t-1}+\boldsymbol{\sigma}_{\zeta} \boldsymbol{\zeta}_{t},
\end{aligned}\right.
$$

where

$$
\begin{aligned}
\theta_{a, t} & =\left(\frac{n_{f, t}}{\theta_{f}}+\frac{n_{c, t}}{\theta_{c}}\right)^{-1}, \quad \mathbf{\Omega}_{a, t}=\frac{1}{\theta_{a, t}}\left(\frac{n_{f, t}}{\theta_{f}} \mathbf{\Omega}_{0}^{-1}+\frac{n_{c, t}}{\theta_{c}}\left(\mathbf{\Omega}_{0}+\lambda \mathbf{V}_{t-1}\right)^{-1}\right)^{-1}, \\
v_{\Delta, t} & :=v_{f, t}-v_{c, t}=\left(\mathbf{z}_{f, t-1}-\frac{\theta_{a, t-1} \mathbf{s}}{\theta_{f}}\right)^{\top}\left(\mathbf{p}_{t}+\mathbf{d}_{t}-R_{f} \mathbf{p}_{t-1}-\frac{\theta_{f}}{2} \mathbf{\Omega}_{0}\left(\mathbf{z}_{f, t-1}+\frac{\theta_{a, t-1} \mathbf{s}}{\theta_{f}}\right)\right) \\
& -\left(\mathbf{z}_{c, t-1}-\frac{\theta_{a, t-1} \mathbf{s}}{\theta_{c}}\right)^{\top}\left(\mathbf{p}_{t}+\mathbf{d}_{t}-R_{f} \mathbf{p}_{t-1}-\frac{\theta_{c}}{2}\left(\mathbf{\Omega}_{0}+\lambda \mathbf{V}_{t-2}\right)\left(\mathbf{z}_{c, t-1}+\frac{\theta_{a, t-1} \mathbf{s}}{\theta_{c}}\right)\right)-C_{\Delta}, \\
n_{c, t} & =1-n_{f, t}, \quad C_{\Delta}=C_{f}-C_{c} \geq 0 .
\end{aligned}
$$

In summary, we have established an adaptively heterogeneous beliefs model of asset prices under the CAPM framework. The resulting model is characterized by a stochastic difference system with seven variables, which is difficult to analyze directly. To understand the interaction of the deterministic dynamics and noise processes, we first study the dynamics of the corresponding deterministic model in Section 3. The stochastic model (2.18) is then analyzed in Section 4 . 


\section{Dynamics of the Deterministic Model}

By assuming that the fundamental price and the dividend are constants $\mathbf{p}_{t}^{*}=$ $\mathbf{p}^{*}, \mathbf{d}_{t}=\overline{\mathbf{d}}$, and there is no supply shock $\boldsymbol{\xi}_{t}=0$, the system (2.18) becomes the deterministic dynamical system 11

$$
\left\{\begin{aligned}
\mathbf{p}_{t}= & \frac{\theta_{a, t}}{R_{f}} \boldsymbol{\Omega}_{a, t}\left[\frac{n_{f, t}}{\theta_{f}} \boldsymbol{\Omega}_{0}^{-1}\left(\mathbf{p}_{t-1}+\boldsymbol{\alpha}\left(\mathbf{p}^{*}-\mathbf{p}_{t-1}\right)\right)\right. \\
& \left.+\frac{n_{c, t}}{\theta_{c}}\left(\boldsymbol{\Omega}_{0}+\lambda \mathbf{V}_{t-1}\right)^{-1}\left(\mathbf{p}_{t-1}+\gamma\left(\mathbf{p}_{t-1}-\mathbf{u}_{t-1}\right)\right)-\mathbf{s}\right]+\frac{1}{R_{f}} \overline{\mathbf{d}} \\
\mathbf{u}_{t}= & \delta \mathbf{u}_{t-1}+(1-\delta) \mathbf{p}_{t}, \\
\mathbf{V}_{t}= & \delta \mathbf{V}_{t-1}+\delta(1-\delta)\left(\mathbf{p}_{t}-\mathbf{u}_{t-1}\right)\left(\mathbf{p}_{t}-\mathbf{u}_{t-1}\right)^{\top}, \\
n_{f, t}= & \frac{1}{1+e^{-\eta v_{\Delta, t-1}}} .
\end{aligned}\right.
$$

The dynamical system (3.1) should not be interpreted as a deterministic approximation of stochastic system (2.18), based on some type of asymptotic convergence, but just as a system obtained by setting the dividend, the supply and the fundamental price at their unconditional mean levels. The analysis of this 'deterministic skeleton' is a common practice in the heterogeneous-agent literature, and it is aimed at gaining some initial insights into the impact of the parameters on the underlying dynamics. Although the properties of the deterministic skeleton do not carry over to the stochastic model in general, important connections between the dynamical structure of the stochastic model and that of the underlying deterministic model exist and have been highlighted in recent literature on stochastic heterogeneous-agent models (see, e.g. Chiarella, He and Zheng (2011) and Zhu, Wang and Guo (2011)).

The system (3.1) has a unique steady state $\left(\mathbf{p}_{t}, \mathbf{u}_{t}, \mathbf{V}_{t}, n_{f, t}\right)=\left(\mathbf{p}^{*}, \mathbf{p}^{*}, \mathbf{0}, n_{f}^{*}\right)$, where, following Eq. (2.12), the fundamental steady state price, $\mathbf{p}^{*}$, is given by

$$
\mathbf{p}^{*}=\frac{1}{r_{f}}\left(\overline{\mathbf{d}}-\theta_{a}^{*} \Omega_{0} \mathbf{s}\right)
$$

\footnotetext{
${ }^{11}$ The state variables $\mathbf{p}_{t}, \mathbf{u}_{t}, \mathbf{V}_{t}$ and $n_{f, t}$ in Eq. (3.1) can be expressed in terms of $\mathbf{p}_{t-1}, \mathbf{u}_{t-1}$, $\mathbf{V}_{t-1}, n_{f, t-1}, \mathbf{p}_{t-2}, \mathbf{u}_{t-2}, \mathbf{V}_{t-2}$ and $n_{f, t-2}$, which have $N, N, N(N+1) / 2,1, N, N, N(N+1) / 2$ and 1 dimensions respectively. So the dimension of the system (3.1) is $N^{2}+5 N+2$. For instance, when $N=1$, it is an 8-dimensional system.
} 
and $n_{f}^{*}=1 /\left(1+e^{\eta C_{\Delta}}\right)$. Hence, at the steady state, $n_{c}^{*}=1-n_{f}^{*}$ and $\theta_{a, t}=\theta_{a}^{*}=$ $1 /\left(n_{f}^{*} / \theta_{f}+n_{c}^{*} / \theta_{c}\right)$. Let $\theta_{0}:=\theta_{f} / \theta_{c}$. Note that if $\theta_{f}=\theta_{c}=\theta$, then $\theta_{a, t}=\theta$ and $\theta_{0}=1$.

For the $N^{2}+5 N+2$ dimensional system (3.1), we are able to obtain the following proposition on the local stability of the steady state. The proof is given in the appendix.

Proposition 3.1. For the system (3.1),

(i) if $R_{f} \geq \delta\left(1+\gamma_{j}\right)$ for all $j \in\{1, \cdots, N\}$, then the steady state $\left(\boldsymbol{p}^{*}, \boldsymbol{p}^{*}, \boldsymbol{O}, n_{f}^{*}\right)$ is locally asymptotically stable;

(ii) if $R_{f}<\delta\left(1+\gamma_{j}\right)$ for all $j \in\{1, \cdots, N\}$, then the steady state is locally asymptotically stable when $C_{\Delta} \neq 0$ and $\eta<\widehat{\eta}_{j}:=\frac{1}{C_{\Delta}} \ln \frac{R_{f}-\delta\left(1-\alpha_{j}\right)}{\theta_{0}\left[\delta\left(1+\gamma_{j}\right)-R_{f}\right]}$ for all $j \in\{1, \cdots, N\}$ and undergoes a Hopf bifurcation when $\eta=\widehat{\eta}_{j}$ for some $j \in\{1, \cdots, N\}$. If $C_{\Delta}=0$, then the steady state is locally asymptotically stable when $\theta_{0} \gamma_{j}<\alpha_{j}+\left(1+\theta_{0}\right)\left(\frac{R_{f}}{\delta}-1\right)$ for all $j$;

(iii) if $R_{f}<\delta\left(1+\gamma_{j}\right)$ for some $j \in J_{o} \subseteq\{1, \cdots, N\}$, then the steady state is locally asymptotically stable when $\eta<\widehat{\eta}_{m}:=\min _{j \in J_{o}} \widehat{\eta}_{j}$ and undergoes a Hopf bifurcation when $\eta=\widehat{\eta}_{m}$.

The results in Proposition 3.1 are significant with respect to the intuitive and simple conditions on the stability of the steady state for such a high dimensional system. First, when the trend followers are not very active (so that $\gamma_{j} \leq R_{f} / \delta-1$ ), the steady state of the system is stable. Second, the stability condition (ii) is equivalent to

$$
\frac{R_{f}}{\delta}-1<\gamma_{j}<\frac{\left(\frac{R_{f}}{\delta}-1\right)\left(1+\theta_{0} e^{\eta C_{\Delta}}\right)+\alpha_{j}}{\theta_{0} e^{\eta C_{\Delta}}}, \quad j=1, \cdots, N .
$$

Hence, even when the trend followers are active (so that $\gamma_{j}>R_{f} / \delta-1$ ), the system can still be stable when the fundamentalists dominate the market at the steady state or, equivalently, the switching intensity is sufficiently small. In short, Proposition 3.1 shows clearly that increases in $C_{c}, \alpha_{j}$ and $\theta_{c}$ stabilize the system, while increases in $C_{f}, \delta, \gamma$ and $\theta_{f}$ destabilize the system. Intuitively, the fundamentalists play a stabilizing role in the market. The activity of fundamentalists is enhanced with 
an increase in $\alpha_{j}$ or decreases in $C_{f}$ (since a decrease in $C_{f}$ increases the market fraction of fundamentalists) and $\theta_{f}$ (since a decrease in $\theta_{f}$ increases fundamentalists' long/short position when the fundamental price moves away from the market price).

To understand how the impact of switching in a market with many risky assets is different from a market with a single risky asset, we consider a special case where agents invest in a market with one risk-free asset and one risky asset, say asset $j$. In this case, system (3.1) reduces to

$$
\left\{\begin{aligned}
p_{j, t}= & \frac{\bar{d}_{j}}{R_{f}}+\frac{1}{R_{f}\left(\frac{n_{f, t}}{\theta_{f} \sigma_{j}^{2}}+\frac{n_{c, t}}{\theta_{c}\left(\sigma_{j}^{2}+\lambda V_{j, t-1}\right)}\right)}\left[\frac{n_{f, t}\left(p_{j, t-1}+\alpha_{j}\left(p_{j}^{*}-p_{j, t-1}\right)\right)}{\theta_{f} \sigma_{j}^{2}}\right. \\
& \left.\quad+\frac{n_{c, t}\left(p_{j, t-1}+\gamma_{j}\left(p_{j, t-1}-u_{j, t-1}\right)\right)}{\theta_{c}\left(\sigma_{j}^{2}+\lambda V_{j, t-1}\right)}-s_{j}\right] \\
u_{j, t}= & \delta u_{j, t-1}+(1-\delta) p_{j, t}, \\
V_{j, t}= & \delta V_{j . t-1}+\delta(1-\delta)\left(p_{j, t}-u_{j, t-1}\right)^{2} \\
n_{f, t}= & \frac{1}{1+e^{-\eta v_{\Delta, t-1}}} .
\end{aligned}\right.
$$

The dynamics of the system (3.3) can be characterized by the following proposition (the proof is given in the appendix).

Proposition 3.2. For the system (3.3),

(i) if $R_{f} \geq \delta\left(1+\gamma_{j}\right)$, then the steady state $\left(p_{j}^{*}, p_{j}^{*}, 0, n_{f}^{*}\right)$ of the system is always locally asymptotically stable;

(ii) if $R_{f}<\delta\left(1+\gamma_{j}\right)$, then the steady state is locally asymptotically stable when $\eta<\widehat{\eta}_{j}$ and $C_{\Delta} \neq 0$ and undergoes a Hopf bifurcation when $\eta=\widehat{\eta}_{j}$. When $C_{\Delta}=0$, the steady state is locally asymptotically stable if $\theta_{0} \gamma_{j}<\alpha_{j}+(1+$ $\left.\theta_{0}\right)\left(\frac{R_{f}}{\delta}-1\right)$.

By comparing the local stability conditions in Propositions 3.1] and 3.2, one can see that the stability conditions of each risky asset due to the increasing switching intensity are independent of the parameters specific to any other asset and, surprisingly, the correlations among risky assets have no impact on the local stability 
properties 12 This result is due to the peculiar properties of the Jacobian matrix and the adaptive behaviour considered (this becomes clear from the proofs in the appendix). Hence the local stability properties of asset $j$ in the multi-asset model are exactly the same as if asset $j$ was considered in isolation (that is, in the model with only risky asset $j$ and the risk-free asset).

Propositions 3.1 and 3.2 provide an initial insight into the mechanisms governing the joint price dynamics of multiple risky assets, showing that locally instability plays a very small role in the spill-over phenomena that we will discuss in what follows, but globally the instability of one asset can spill over to the other assets due to its correlations with other assets.

To better understand the implications of Propositions 3.1 and 3.2 and the price dynamics of the model, we consider an example of two risky assets and a riskless asset with

$$
\boldsymbol{\Omega}_{0}=\left(\begin{array}{cc}
\sigma_{1}^{2} & \rho_{12} \sigma_{1} \sigma_{2} \\
\rho_{12} \sigma_{1} \sigma_{2} & \sigma_{2}^{2}
\end{array}\right)
$$

and set $13 \mathrm{~s}=(0.1,0.1)^{\top}, \theta_{f}=1, \theta_{c}=1, \lambda=1.5, C_{f}=4, C_{c}=1, \rho_{12}=0.5$, $\delta=0.98, \boldsymbol{\gamma}=\operatorname{diag}[0.3,0.3]$ and $\boldsymbol{\alpha}=\operatorname{diag}[0.4,0.5]$. We choose the annual values 14 of the following parameters: $r_{f}=0.025, \sigma_{1}=0.6, \sigma_{2}=0.4$ and $\overline{\mathbf{d}}=(0.08,0.05)^{\top}$. In this paper, we consider monthly time steps (i.e. $K=12$ ).

Based on this set of parameters, the model has the following implications. First, the equilibrium steady state fundamental price decreases when the correlation coefficient increases and, when the fundamental strategy costs more than the trend follower strategy, the steady state market fraction of the fundamentalists reduces as the switching intensity increases. These results are illustrated in Figs. 3.1 (a) and

\footnotetext{
${ }^{12}$ Mathematically, this is due to the fact that the fitness measure and the variance-covariance matrices are in higher order terms. Certainly they can affect the nonlinear dynamics, but not the dynamics of the linearized system.

${ }^{13}$ The set of parameters is fixed in all numerical analysis unless specified otherwise.

${ }^{14}$ The annualised parameters are converted to monthly, weekly and daily parameters in the standard way, by rescaling $r_{f}, \boldsymbol{\Omega}_{0}, \boldsymbol{\alpha}, \overline{\mathbf{d}}, \boldsymbol{\gamma}, C_{f}$ and $C_{c}$ via the factor $1 / K$, where the frequency $K$ is set to 12 (monthly), 50 (weekly), 250 (daily). As shown in Chiarella et al. (2012), the parameter $\delta$ is converted to $K \delta /[1+(K-1) \delta]$ to preserve the average memory length of the time average of past returns.
} 


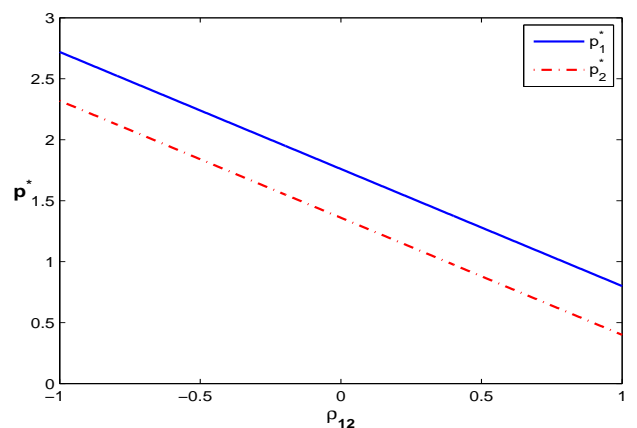

(a) Steady-state prices

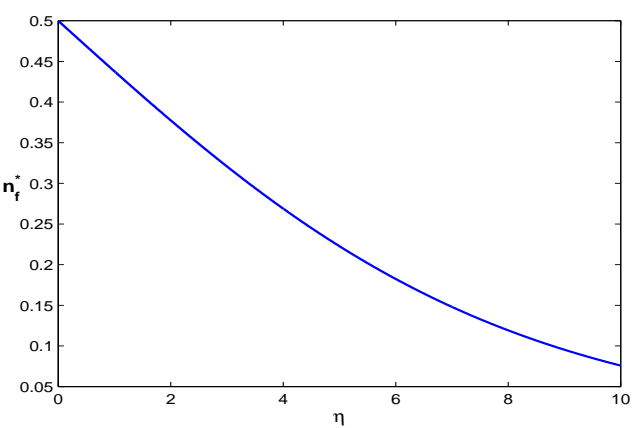

(b) Steady-state market fraction

Figure 3.1. (a) The fundamental steady state price $\mathbf{p}^{*}=\left(p_{1}^{*}, p_{2}^{*}\right)$ as a function of the correlation $\rho_{12}$ with $\eta=1$; (b) The equilibrium market fractions of fundamentalists $n_{f}^{*}$ as a function of the switching intensity $\eta$.

(b) respectively for the two-asset system (3.1). In fact, Eq. (3.2) determines the dependence of the steady state fundamental price on the parameters. Fig. 3.1 (a) illustrates a negative linear relationship between the fundamental steady state price $\mathbf{p}^{*}=\left(p_{1}^{*}, p_{2}^{*}\right)$ and the correlation $\rho_{12}$.

Secondly, as implied by Propositions 3.1 and 3.2, asset prices become unstable as the switching intensity $\eta$ increases. This is illustrated in Fig. 3.2. With the chosen parameters, one can verify that $R_{f}<\delta\left(1+\gamma_{j}\right)$ for $j=1,2$, and the bifurcation values for asset one and two are $\widehat{\eta}_{1} \approx 2.2384$ and $\widehat{\eta}_{2} \approx 3.0485$ respectively. According to Proposition 3.1, when $\eta<\widehat{\eta}_{1}$ the two prices are stable; when $\widehat{\eta}_{1}<\eta<\widehat{\eta}_{2}$, the price of asset two is still stable, however the price of asset one becomes unstable; when $\eta>\widehat{\eta}_{2}$, the prices of both assets become unstable 15 Fig. 3.2 plots the price bifurcation diagrams with respect to the switching intensity parameter $\eta$ for both system (3.1) with two risky assets and system (3.3) with one risky asset. For the single risky asset model, Figs. 3.2 (c) and (d) show that an increase in the switching intensity $\eta$ makes the steady state price unstable, leading to the complicated price dynamics documented in Brock and Hommes (1998). With two risky assets, Figs.

\footnotetext{
${ }^{15}$ Note that here we use the words 'stable' and 'unstable' in a loose, yet intuitive, sense. Strictly speaking, the local asymptotic stability of the steady state of the multi-asset model is lost when $\eta=\min _{j} \widehat{\eta}_{j}$, as stated in Proposition 3.1.
} 


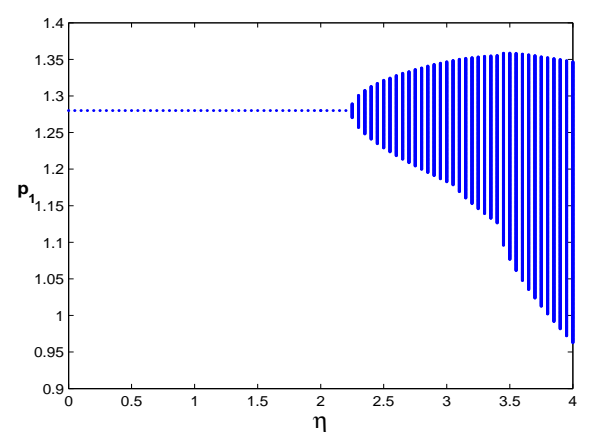

(a) Asset one price in the two-asset model

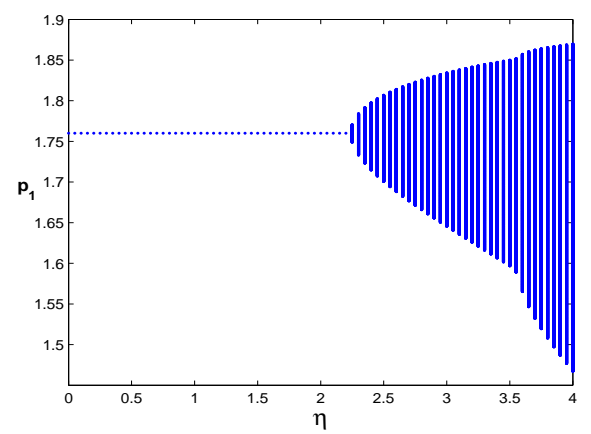

(c) Price in a single asset one model

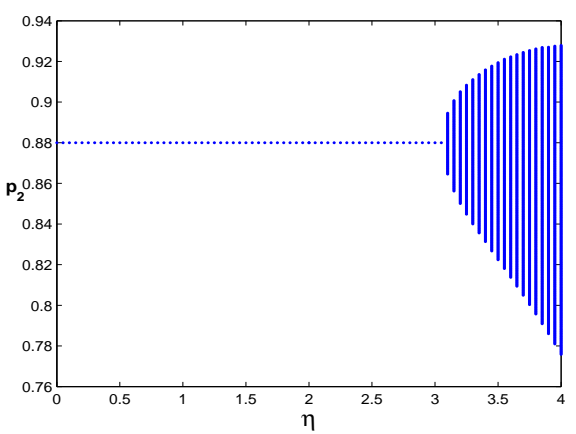

(b) Asset two price in the two-asset model

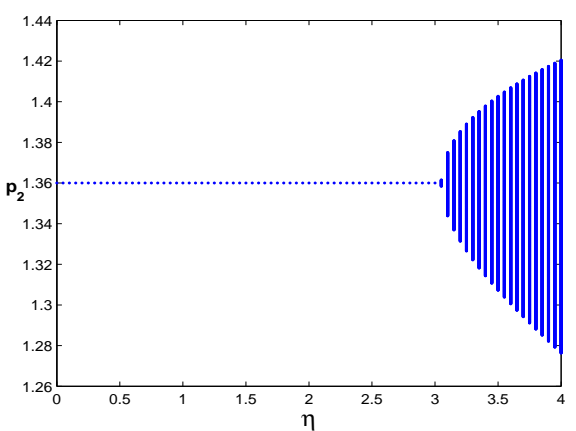

(d) Price in a single asset two model

FiguRE 3.2. The bifurcations of the two risky asset prices with respect to $\eta$ (a) and (b), the two asset model (3.1); and (c) and (d) the single risky asset model (3.3).

3.2 (a) and (b) show that the steady state is stable when the switching intensity $\eta$ is low, but becomes unstable as the switching intensity increases. The time series of prices and the market fraction of the fundamentalist in Figs. 3.3 (a)-(c) provide further evidence on the analysis above. At first, both assets and the market fraction are stable and constant when $\eta=1.5$ is small (Fig. 3.3 (a)). As $\eta$ increases to 2.5, asset one and then asset two become unstable as illustrated in Fig. 3.3 (b). As $\eta$ increases to 3.5, both asset prices become unstable (see Fig. 3.3 (c)). Also the price of risky asset one of the two asset model is more irregular compared to the regular fluctuations in Fig. 3.3 (f) of the one asset model. Also, as the switching intensity $\eta$ increases, even small fluctuations in the market fractions of agents can cause large fluctuations in asset prices. 


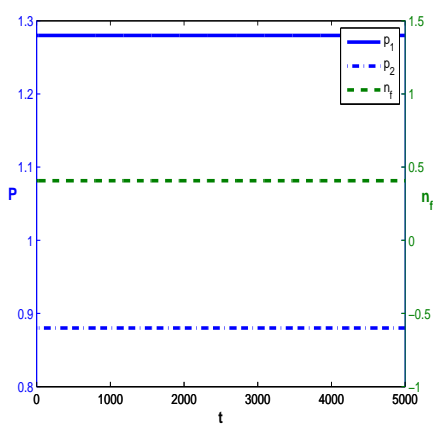

(a)

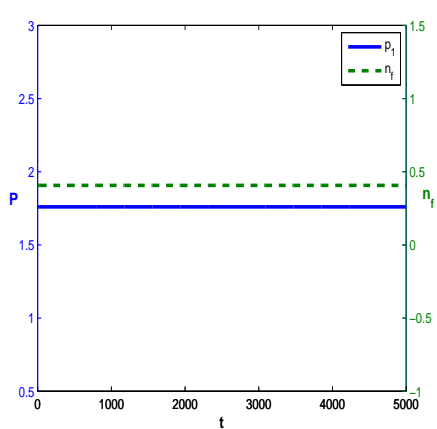

(d)

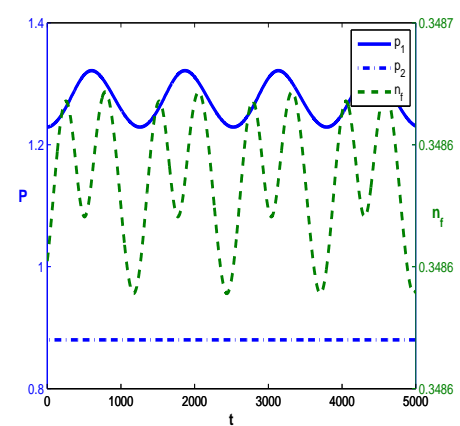

(b)

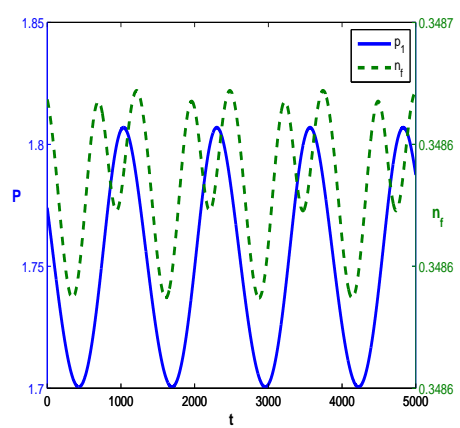

(e)

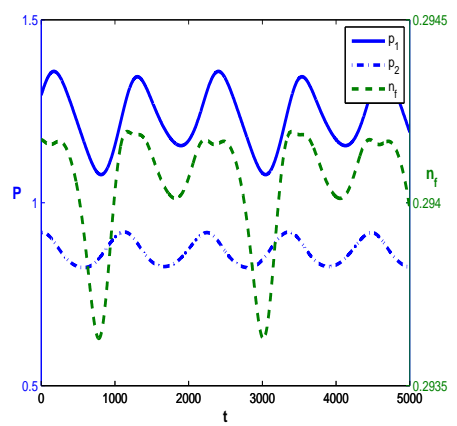

(c)

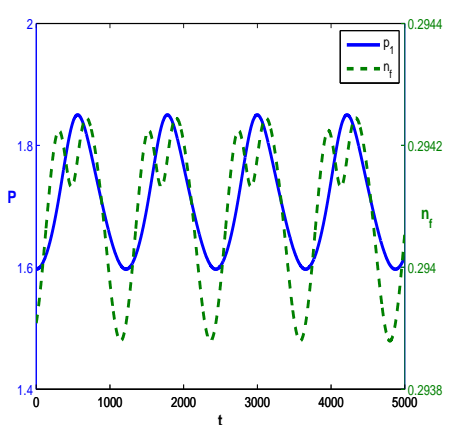

(f)

Figure 3.3. Time series plots of $p_{1, t}, p_{2, t}$ and $n_{f, t}$ in the two-asset model ((a)-(c)) and of $p_{1, t}$ in the single-asset one model ((d)-(f)) with $\eta=1.5$ in (a) and (d); $\eta=2.5$ in (b) and (e); and $\eta=3.5$ in (c) and (f).

Thirdly, the model displays a very interesting spill-over effect, which can be very different from portfolio effect. As we discussed earlier, the stability is a local result and the stability conditions of the risky assets are independent among the risky assets. When one asset becomes unstable, one would expect the spill-over of instability of the asset to spread to the other assets due to the portfolio effect. However, this may not always be the case, as demonstrated in Fig. 3.4. For $\eta=2.5$, Fig. 3.4 (a) shows that the price is unstable for asset one, but stable for asset two. Intuitively, the price fluctuations in asset one would be caused by changing portfolio positions taken by the two types of agents for asset one, which is confirmed by Fig. 3.4 (c). However, this intuition does not carry over to asset two for which the price is constant but the portfolio positions taken by agents for the asset are also varying, 


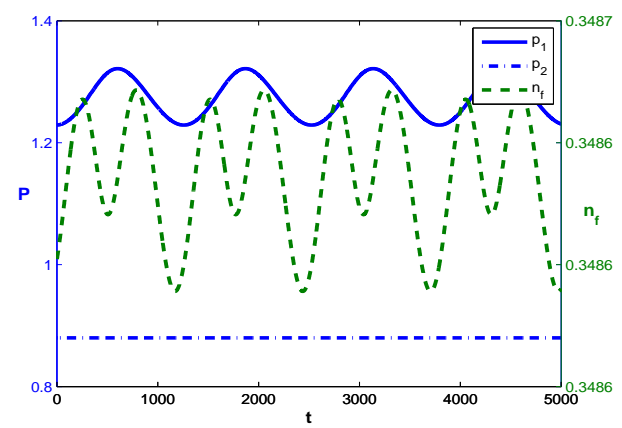

(a) Prices

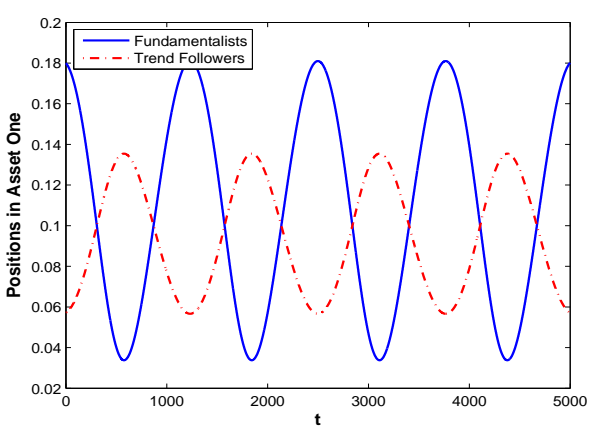

(c) Positions in Asset One

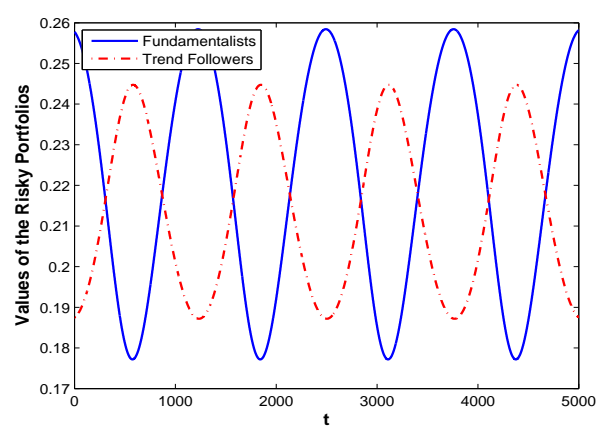

(b) Values of the risky portfolios

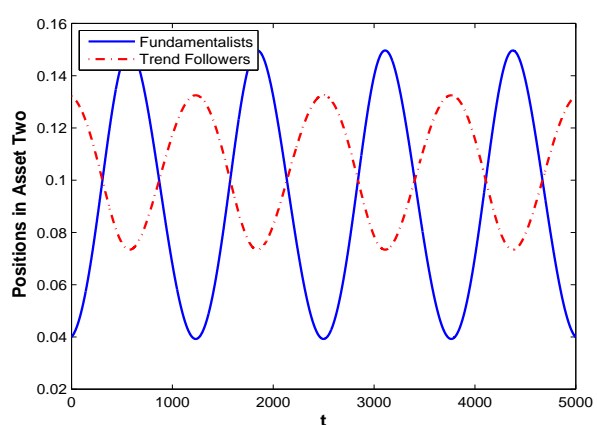

(d) Positions in Asset Two

FigurE 3.4. The time series of (a) the prices and market fraction; (b) portfolio values of the fundamentalists and trend followers; and portfolio positions of two types of agents in asset one (c) and asset two (d). Here $\eta=2.5$.

as illustrated in Fig. 3.4 (d). The portfolio variations due to the portfolio effect lead to the fluctuations of the portfolio values of the agents that are illustrated in Fig. 3.4 (b). How can this happen? As a matter of fact, there are two reasons behind this interesting phenomena. First, the variations of the risky assets in the portfolios are caused by the correlation between risky assets (reflected in both $\Omega_{o}$ and $\mathbf{V}_{t}$ ) and the time-varying population fractions. Hence, even when the price of asset two is constant, the portfolio positions of agents in asset two may not be constant. Secondly, the spill-over effect is a nonlinear rather than a linear effect, meaning that the stability of asset two in the nonlinear system is observed when the initial values are near the steady state values; otherwise, stability may not be maintained. Fig. 3.5 shows how the instability of asset one spill over to asset two 
for $\eta \in\left(\hat{\eta}_{1}, \hat{\eta}_{2}\right)$ increases from 2.24 in (a) to 2.5 in (b) and then to 3.04 in (c) when the initial prices are far away from the steady state price levels. Note that asset two is locally stable for $\eta \in\left(\hat{\eta}_{1}, \hat{\eta}_{2}\right)$. Hence the spill-over effect reflects the dynamics of the nonlinear system. In fact, we do observe such spill-over effects in the bifurcation plots in Fig. 3.2. Note that the first small price jump of asset one in Fig. 3.2 (a) after the initial bifurcation (at $\eta=\hat{\eta}_{1}$ ) occurs at $\eta=\hat{\eta}_{2}$, which is the bifurcation of the second asset in the single asset model (in Fig. 3.2 (d)). This implies that, when asset two becomes unstable, there is a spill-over effect from asset two to asset one characterized by the price jump of asset one near $\hat{\eta}_{2}$. Interestingly, as the intensity increases further, say $\eta \approx 3.4$, the fluctuations of asset one increases significantly, which is demonstrated by the large price jump of asset one for $\eta>3.4$.

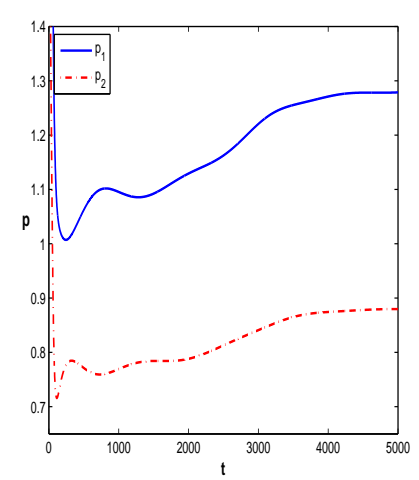

(a) $\eta=2.24$

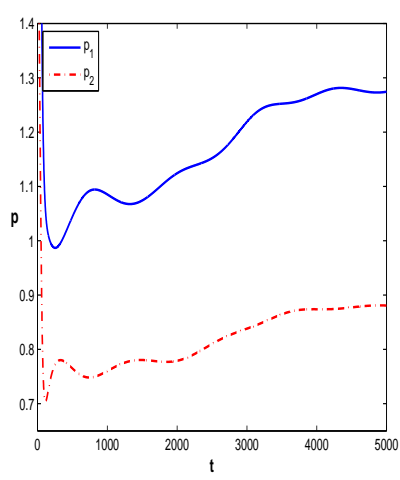

(b) $\eta=2.5$

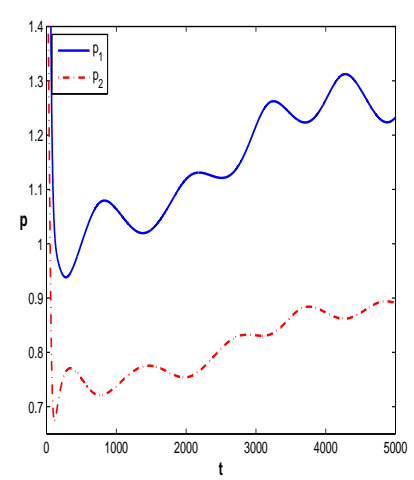

(c) $\eta=3.04$

Figure 3.5. The market prices for $\eta=2.24$ (a), 2.5 (b) and 3.04 (c) when initial values are far away from the steady state.

In addition, numerical simulations (not reported here) show that an increase in $\rho_{12}$ leads to increases in the fluctuations of the prices when the system (3.1) becomes unstable. Intuitively, as the correlation of the two risky assets increases, diversification becomes less effective, hence assets become more risky, and consequently the fundamental equilibrium prices decrease (in order to have a high expected return). Also, simulations show (not reported here) that market prices become more volatile when the trend followers are less concerned about the sample variance; that is when $\lambda$ becomes small, even though $\lambda$ does not affect the local stability of the system 
(3.1). In fact, when $\lambda$ becomes small, the demand of the trend followers increases so that they become more active in the market, leading to a more volatile market.

In summary, we have shown that the rational behaviour of agents in switching to better performing strategies can lead to market instability and a non-linear spillover of price fluctuations from one asset to other assets. The nonlinear dynamics due to the spill-over effect can lead to high trading volume and high volatility. This becomes clearer in the discussion of the stochastic model in the next section.

\section{Price Behaviour of the Stochastic Model}

In this section, through numerical simulations, we first focus on the spill-over effect by examining the interaction between the dynamics of the deterministic model and the noise processes and explore the potential power of the model to explain price deviations from the fundamental prices and also high volatility. We then provide an evolutionary capital asset pricing model (ECAPM) and compare the ex-ante betas with the rolling window estimates of the betas used in the literature. Finally we study the relationship between the price volatility and trading volumes. We choose $\boldsymbol{\sigma}_{\kappa}=\operatorname{diag}[0.001,0.001]$ and $\boldsymbol{\sigma}_{\zeta}=\operatorname{diag}[0.002,0.002]$, representing $0.1 \%$ and $0.2 \%$ standard deviations of the noisy supply and dividend processes respectively in this section.

4.1. The Spill-over Effect. First, we examine the spill-over effect by exploring the joint impact of the switching intensity $\eta$ and the two noise processes on the market price dynamics. To examine the impact of stability of the deterministic model on the price dynamics, in particular, the time-varying betas, for the stochastic model, with the same random draws of the dividend and supply noise processes, Fig. 4.1 plots the fundamental price (the blue and green dotted lines) and the market prices (the red and black solid lines) in (a) and (b) and the corresponding market fractions of the fundamentalists of the two-asset model for two different switching intensities $\eta$. For $\eta=1.5$, Figs. 4.1 (a) and (c) demonstrate that the market price follows the fundamental price closely with about $40 \%$ of the fundamentalists. This is underlined by the stable fundamental steady state of the deterministic model (3.1) illustrated in Fig. 3.3 (a). For $\eta=3.5$, Figs. 4.1 (b) and (d) indicate that the market price 


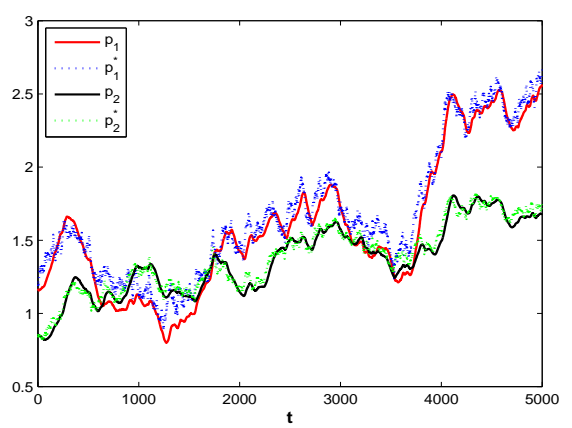

(a) Prices with $\eta=1.5$

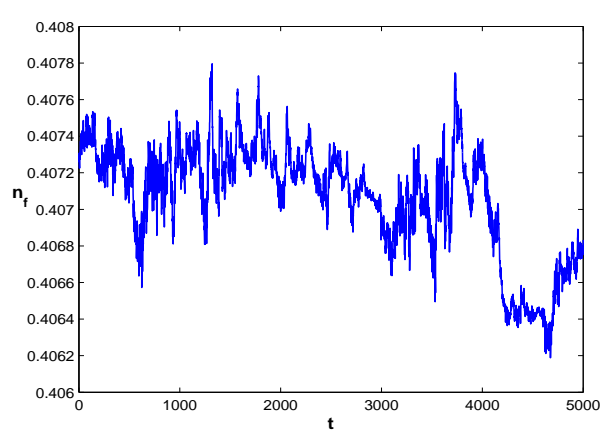

(c) Market fraction with $\eta=1.5$

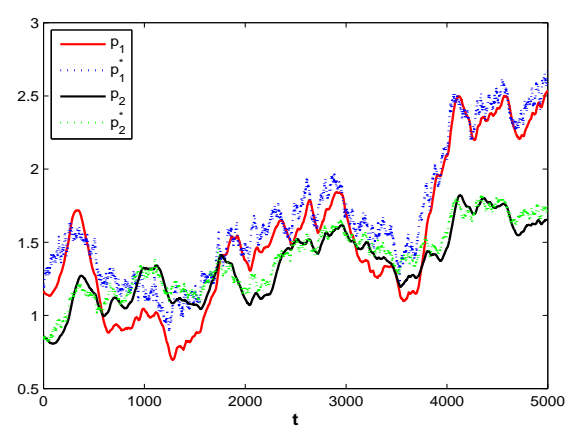

(b) Prices with $\eta=3.5$

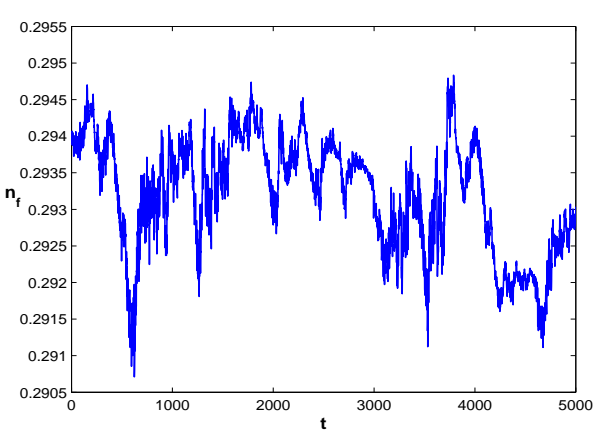

(d) Market fraction with $\eta=3.5$

Figure 4.1. The time series of the fundamental price (the dotted line) and the market prices (the solid line) of the two-asset model with (a) $\eta=1.5$ and (b) $\eta=3.5$, and the corresponding market fractions of the fundamentalists in (c) and (d).

fluctuates around the fundamental price in a cyclical fashion with about $29 \%$ of the fundamentalists, which is underlined by the bifurcation of periodic oscillations of the corresponding deterministic model (see Fig. 3.3 (c)). Corresponding to Fig. 3.4 for the deterministic model, Fig. 4.2 plots the time series of the prices in (a), the market fraction of the fundamentalists in (b), the portfolio values of the two agents in (c), the portfolio positions in asset one (d) and asset two (e) of the stochastic model. The large fluctuations of the stochastic model, in particular in the portfolio values and the portfolio positions, compared to the deterministic model reflect the impact of the nonlinear interaction of the spill-over effects and the noise processes. 


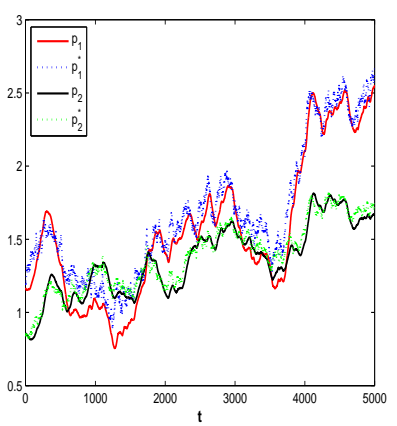

(a) Prices

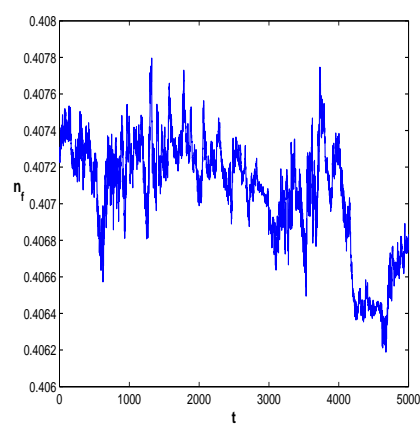

(b) Market fractions

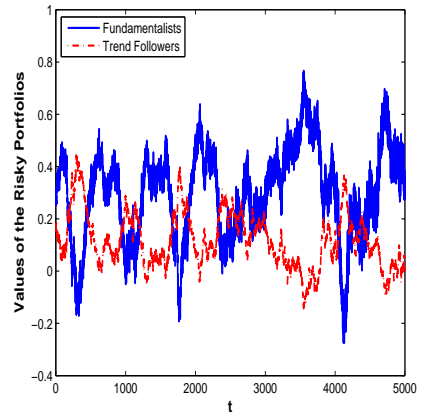

(c) Portfolio values

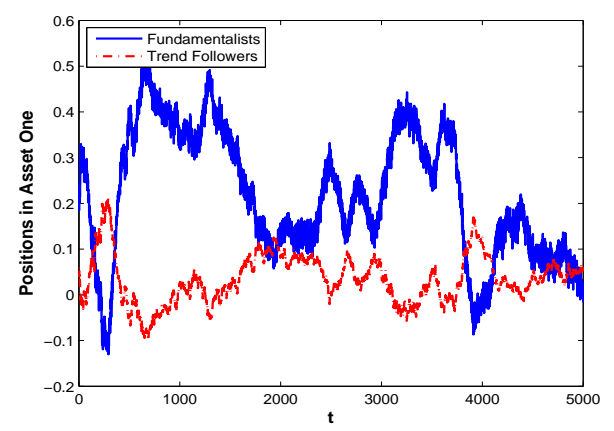

(d) Positions in asset One

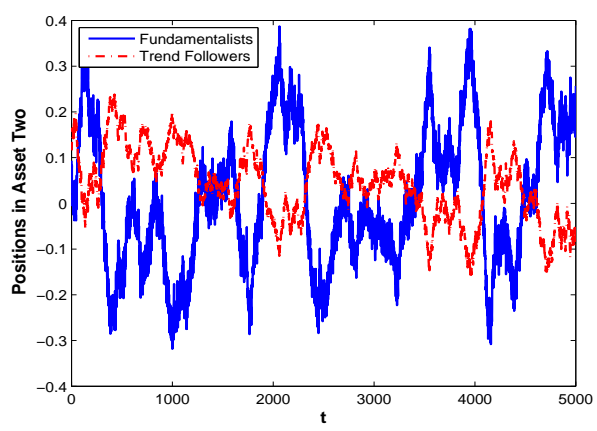

(e) Positions in asset Two

FigurE 4.2. The time series of prices (a), market fractions of the fundamentalists (b), the portfolio values of two types of agents (c), the portfolio positions in asset one (d) and asset two (e) of the stochastic model with $\eta=2.5$.

4.2. Time-varying Betas. Next, we examine the stochastic nature of the timevarying beta coefficients of the evolutionary CAPM. The value of the market portfolio $\mathbf{s}$ at time $t$ in the market equilibrium is given by $W_{m, t}=\mathbf{p}_{t}^{\top} \mathbf{s}$ and the payoff is $W_{m, t+1}=\mathbf{x}_{t+1}^{\top} \mathbf{s}$. Hence, under the consensus belief there holds

$$
E_{a, t}\left(W_{m, t+1}\right)=E_{a, t}\left(\mathbf{x}_{t+1}\right)^{\top} \mathbf{s}, \quad \operatorname{Var}_{a, t}\left(W_{m, t+1}\right)=\mathbf{s}^{\top} \Omega_{a, t} \mathbf{s}
$$

Define the returns of risky asset $j$ and the market portfolio $m$, respectively, by

$$
r_{j, t+1}=\frac{x_{j, t+1}}{p_{j, t}}-1, \quad r_{m, t+1}=\frac{W_{m, t+1}}{W_{m, t}}-1
$$




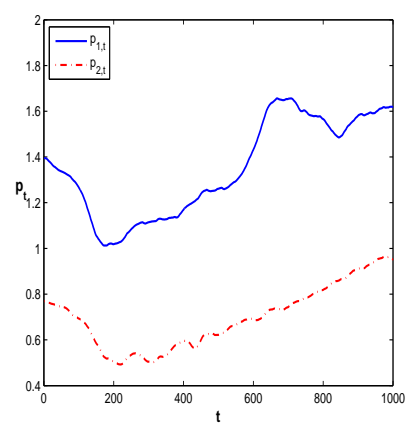

(a) Prices

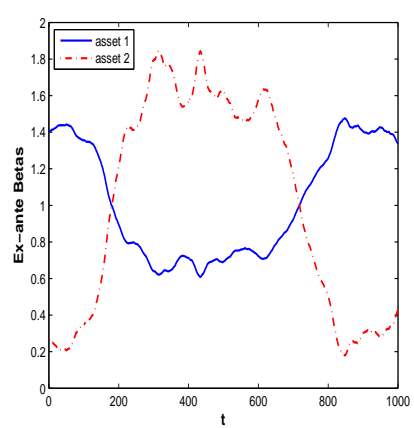

(d) Ex-ante betas

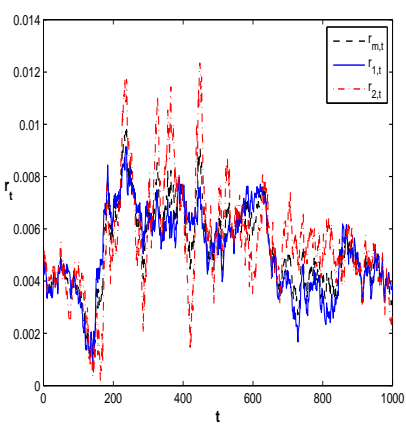

(b) Returns

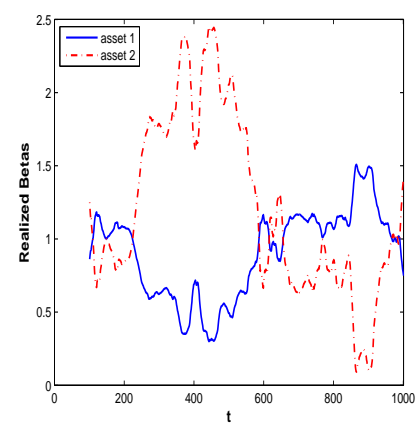

(e) Rolling estimates of betas with rolling window of 100

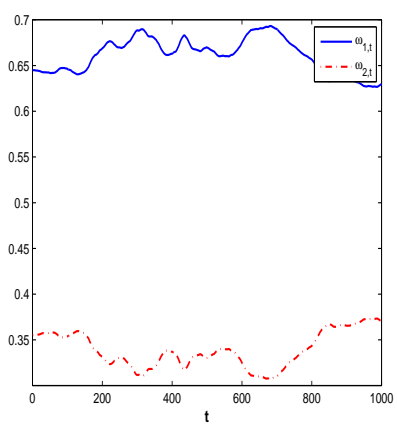

(c) Market portfolio

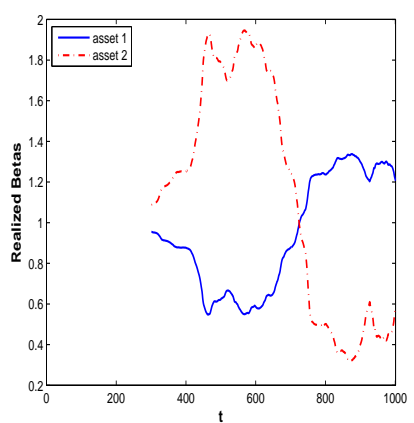

(f) Rolling estimates of betas with rolling window of 300

FigurE 4.3. Time series of (a) the market prices; (b) the returns; (c) the proportions of the total market wealth invested in risky assets; (d) the ex-ante betas of the risky assets; and the estimates of the betas using rolling windows of (e) 100 and (f) 300 with $\eta=1.5$.

from which

$$
E_{a, t}\left(r_{j, t+1}\right)=\frac{E_{a, t}\left(x_{j, t+1}\right)}{p_{j, t}}-1, \quad E_{a, t}\left(r_{m, t+1}\right)=\frac{E_{a, t}\left(W_{m, t+1}\right)}{W_{m, t}}-1 .
$$

Following Chiarella et al. (2011), we obtain the standard CAPM-like return relation

$$
E_{a, t}\left(\mathbf{r}_{t+1}\right)-r_{f} \mathbf{1}=\boldsymbol{\beta}_{a, t}\left[E_{a, t}\left(r_{m, t+1}\right)-r_{f}\right]
$$

where

$$
\boldsymbol{\beta}_{a, t}=\left(\beta_{1, t}, \cdots, \beta_{N, t}\right)^{\top}, \quad \beta_{j, t}=\frac{\operatorname{Cov}_{a, t}\left(r_{m, t+1}, r_{j, t+1}\right)}{\operatorname{Var}_{a, t}\left(r_{m, t+1}\right)}
$$

are the beta coefficients in market equilibrium. Notice that the betas in Eq. (4.4) are ex-ante in the sense that they reflect the market equilibrium condition under the 
consensus belief $E_{a, t}$ and $\boldsymbol{\Omega}_{a, t}$. In addition, Eq. (4.2) also implies $r_{m, t+1}=\boldsymbol{\omega}_{t}^{\top} \mathbf{r}_{t+1}$, leading to $\boldsymbol{\omega}_{t}^{\top} \boldsymbol{\beta}_{a, t}=1$, where $\boldsymbol{\omega}_{t}=\mathbf{P}_{t} \mathbf{s} /\left(\mathbf{p}_{t}^{\top} \mathbf{s}\right)$ with $\mathbf{P}_{t}=\operatorname{diag}\left[p_{1, t}, \cdots, p_{N, t}\right]$ are the proportions of the total wealth (ex dividend) in the economy invested in the risky assets at time $t$.

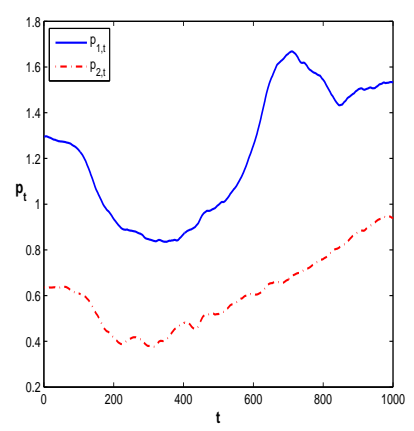

(a) Prices

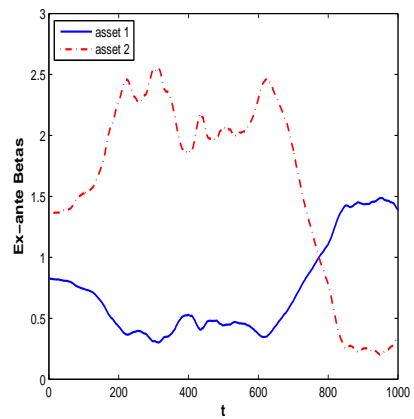

(d) Ex-ante betas

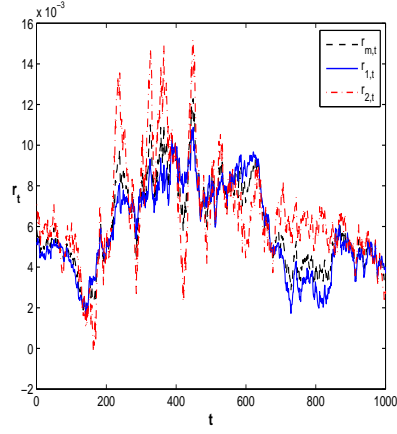

(b) Returns

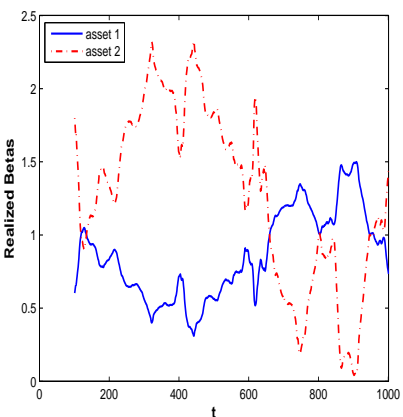

(e) Rolling estimates of betas with rolling window of 100

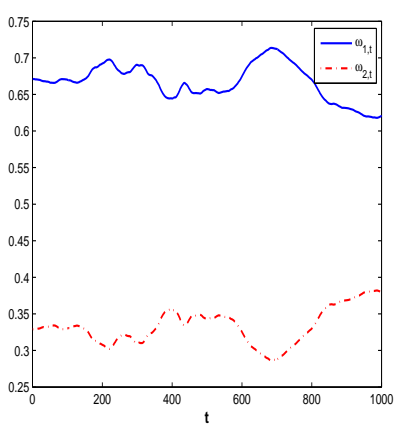

(c) Market portfolio

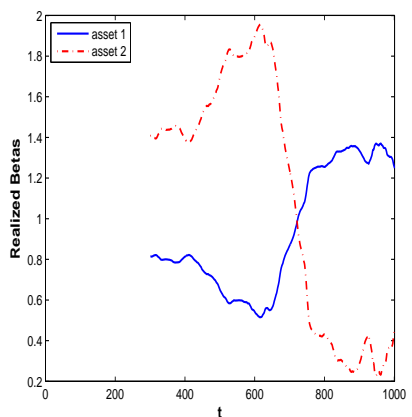

(f) Rolling estimates of betas with rolling window of 300

FiguRE 4.4. Time series of (a) the market prices; (b) the returns; (c) the proportions of the total market wealth invested in risky assets; (d) the ex-ante betas of the risky assets; and the estimates of the betas using rolling windows of (e) 100 and (f) 300 with $\eta=3.5$.

To examine the time-varying betas of the stochastic model, we choose two switching intensities $\eta=1.5$ and 3.5 as before. The time series of the market prices, fractions of the fundamentalists, the proportions of the market portfolio invested in the two risky assets, the ex-ante betas of the risky assets, and the estimates of the betas using rolling windows of 100 and 300 are illustrated in Fig. 4.3 for $\eta=1.5$ and in Fig. 4.4 for $\eta=3.5$. For $\eta=1.5$, the fundamental price of the deterministic 
model is stable, the variation of the beta coefficients in Fig. 4.3 (d) is large but less significant compared to the beta coefficients in Fig. 4.4 (d) for $\eta=3.5$ (where the fundamental price of the deterministic model is unstable). Both the pattern and the level of the beta coefficients for $\eta=1.5$ are very different from those for $\eta=3.5$. More importantly, both Figs. 4.3 and 4.4 show that the rolling estimates of the betas do not necessarily reflect the nature of the ex-ante betas implied by the CAPM, which is consistent with the results in Chiarella et al. (2012). Interestingly, the estimated betas for window of 100 are more volatile compared to the ex-ante betas. However, an increase in rolling window from 100 to 300 in (e) and (f) of Figs. 4.3 and 4.4 smooths the variations of the beta estimates significantly, leading to a similar pattern to the ex-ante betas.

4.3. Trading Volume and Volatility. Finally, we examine the dynamic relation between price volatility and trading volume. As in Banerjee and Kremer (2010), the price volatility is measured by the price difference $\left|p_{j, t}-p_{j, t-1}\right|$ and the trading volume at time $t$ is defined by

$$
\begin{aligned}
\mathbf{X}_{t}=\min & \left\{n_{f, t-1}, n_{f, t}\right\}\left|\mathbf{z}_{f, t}-\mathbf{z}_{f, t-1}\right|+\min \left\{n_{c, t-1}, n_{c, t}\right\}\left|\mathbf{z}_{c, t}-\mathbf{z}_{c, t-1}\right| \\
& +\left|n_{f, t}-n_{f, t-1}\right| \hat{\mathbf{X}}_{t},
\end{aligned}
$$

where

$$
\hat{\mathbf{X}}_{t}= \begin{cases}\left|\mathbf{z}_{f, t}-\mathbf{z}_{c, t-1}\right|, & n_{f, t} \geq n_{f, t-1} \\ \left|\mathbf{z}_{c, t}-\mathbf{z}_{f, t-1}\right|, & n_{f, t}<n_{f, t-1}\end{cases}
$$

Due to the switching mechanism, the total trading volume $\mathbf{X}_{t}$ in (4.5) can be decomposed into three components. The first and second components correspond to the trading volume of the agents who use, respectively, the fundamental and trend following trading strategies at both time $t-1$ and $t$. The third component corresponds to the trading volume of those agents who change their strategies from $t-1$ to $t$. In particular, when $n_{f, t}>n_{f, t-1}$, a fraction of $n_{f, t}-n_{f, t-1}$ agents change their strategies from the trend following strategy at time $t-1$ (with a demand of $z_{c, t-1}$ ) to the fundamental strategy at time $t$ (with a demand of $z_{f, t}$ ). 


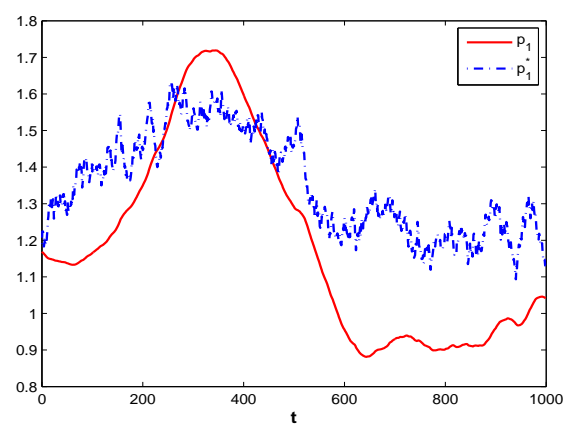

(a) Prices of asset one

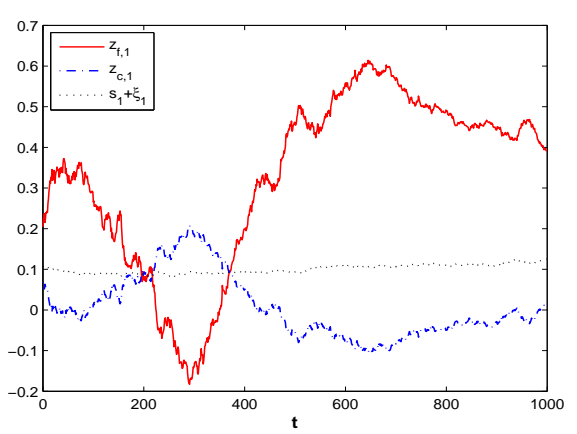

(c) The positions in asset one

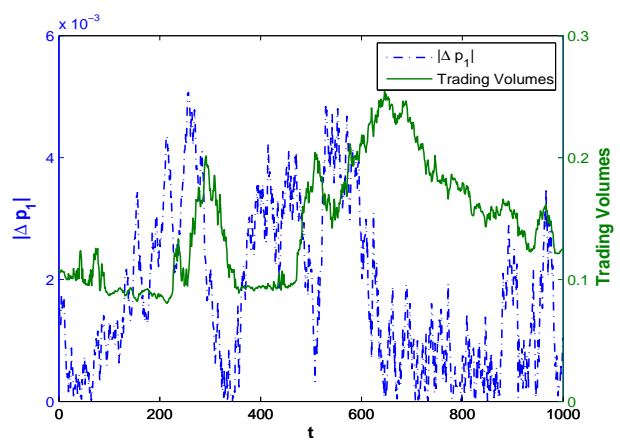

(e) Volatility and volumes of asset one

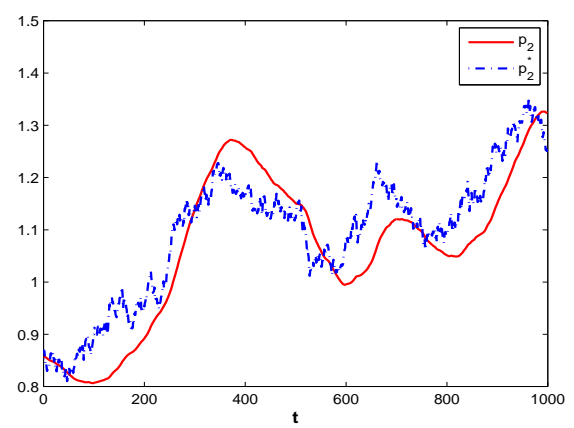

(b) Prices of asset two

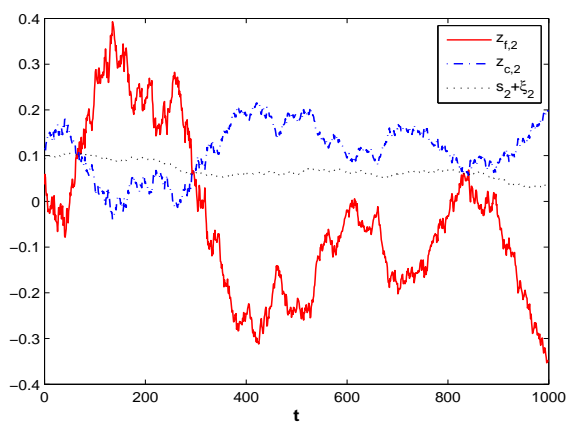

(d) The positions in asset two

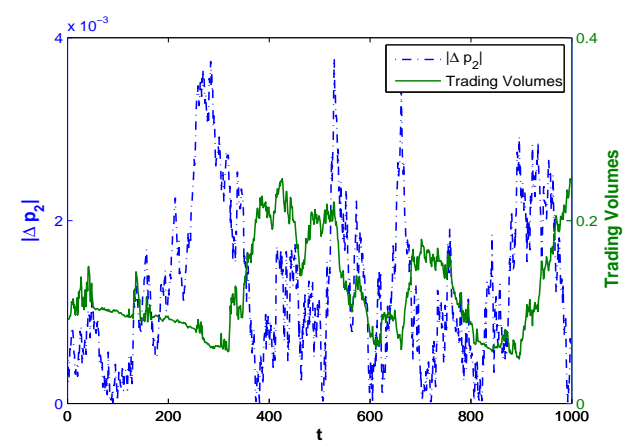

(f) Volatility and volumes of asset two

FiguRE 4.5. The time series of market prices and fundamental price of asset one (a) and asset two (b); the portfolio positions in asset one (c) and asset two (d); the price volatility and trading volume of asset one (e) and asset two (f). Here $\rho_{12}=0.5$ and $\eta=1.5$.

To explore the dynamics of the volatility and trading volume, we set $\eta=1.5$ and choose two values of $\rho_{12}=0.5$ and -0.9 to examine the impact of the correlation. The time series of prices, demands, price volatility and trading volumes are 


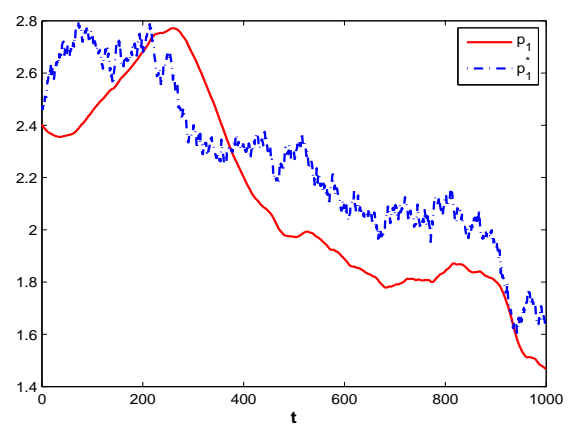

(a) Prices of asset one

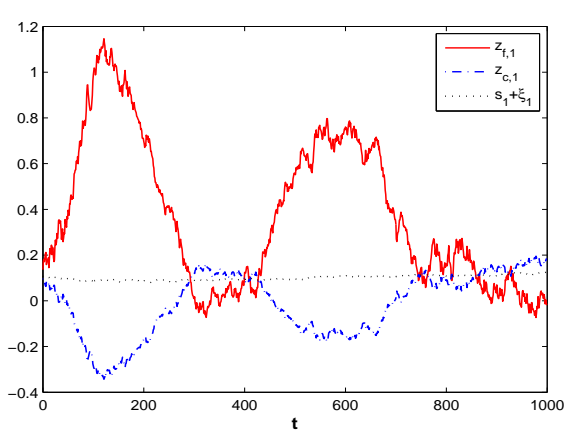

(c) The positions in asset one

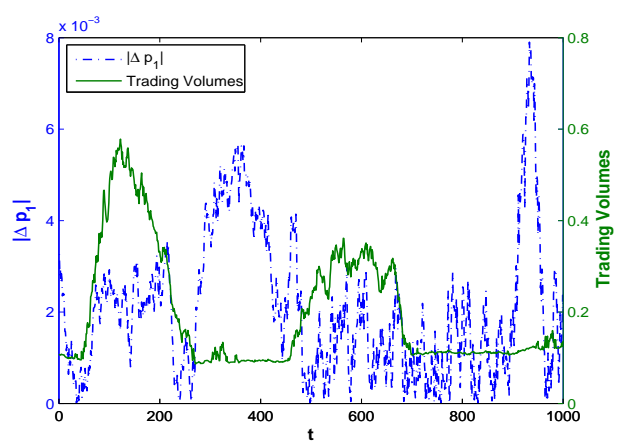

(e) Volatility and volumes of asset one

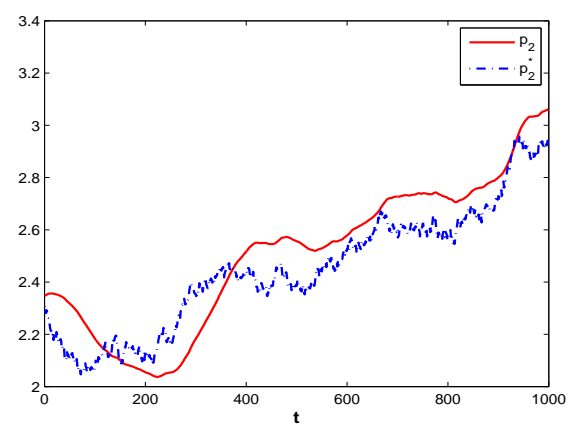

(b) Prices of asset two

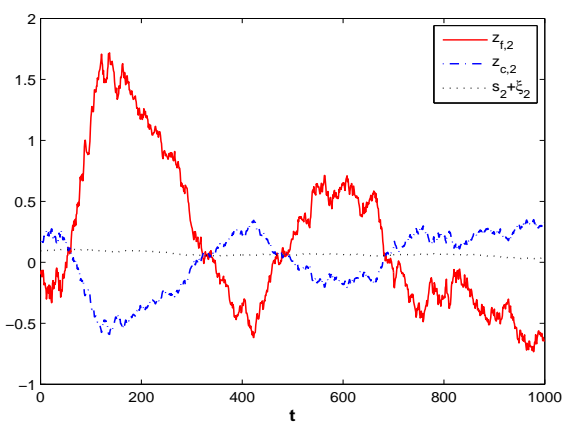

(d) The positions in asset two

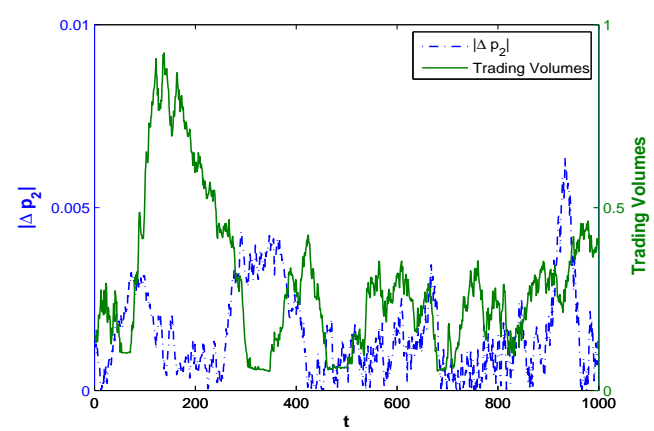

(f) Volatility and volumes of asset two

FiguRE 4.6. The time series of market prices and fundamental price of asset one (a) and asset two (b); the portfolio positions in asset one (c) and asset two (d); the price volatility and trading volume of asset one (e) and asset two (f). Here $\rho_{12}=-0.9$ and $\eta=1.5$.

illustrated in Fig. 4.5 with $\rho_{12}=0.5$ and Fig. 4.6 with $\rho_{12}=-0.9$ for a typical simulation. With the same random seeds, Figs. 4.5 and 4.6 illustrate the significant impact of the portfolio effect due to the different choices of the correlation coefficient 


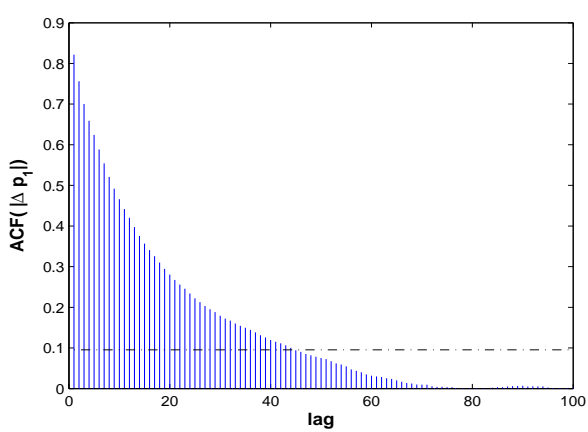

(a) The ACs of the price volatility

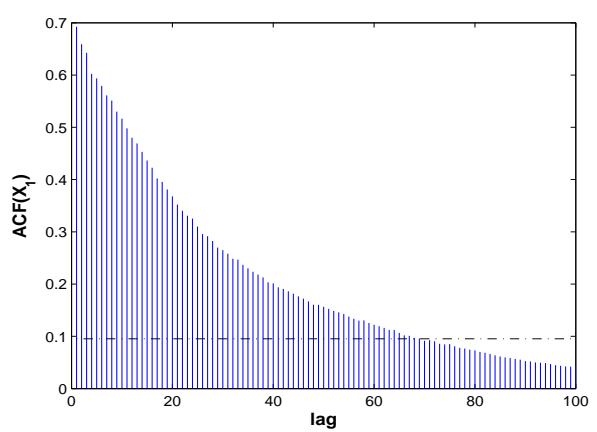

(b) The ACs of the trading volume

FigurE 4.7. The ACs of the price volatility and the trading volume of asset one with $\eta=1.5$.

$\rho_{12}=0.5$ and -0.9 . Figs. 4.5 and 4.6 (a) and (b) show that the market prices can deviate from the fundamental prices from time to time, though they follow each other in the long-run. Figs. 4.5 and 4.6 (c) and (d) show that the fundamentalists and trend followers take opposite positions in risky assets in general, as expected in market equilibrium with two types of agents trading against each other. Figs. 4.5 and 4.6 (e) and (f) indicate that both volatility and trading volume are persistent, which is further verified by the autocorrelations (ACs) of the price volatility and trading volume of risky asset one in Figs. 4.7 (a) and (b) respectively. The results are based on 100 numerical simulations with the same parameters but different random processes. They demonstrate that the ACs for both the volatility and trading volume are highly significant and decaying over long lags.

Intuitively, the correlation should play an important role in the relation between volatility and trading volume. With the two different values of $\rho_{12}=0.5$ and -0.9 , Figs. 4.5 and 4.6 (e) and (f) depict the relationship between the price volatility and the trading volume of the two assets model. The observation is summarized statistically by the plot in Fig. 4.8 of the relation between the correlation coefficient $\rho_{12}$ and the average correlation between price volatility and trading volume of the two assets (asset one in (a) and asset two in (b)) and correlation in volatility (c) and trading volumes among the two assets based on 100 simulations. We observe that the correlation between the volatility and trading volume is positive (negative) when assets are less (more) correlated, but the correlations in both volatilities and 


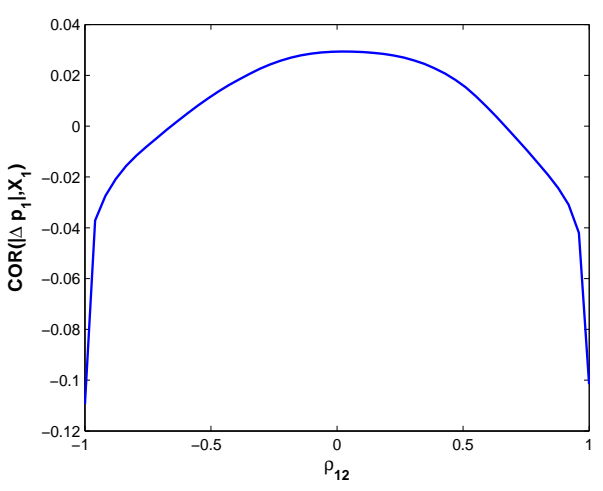

(a) Correlation between trading volume and volatility for asset one

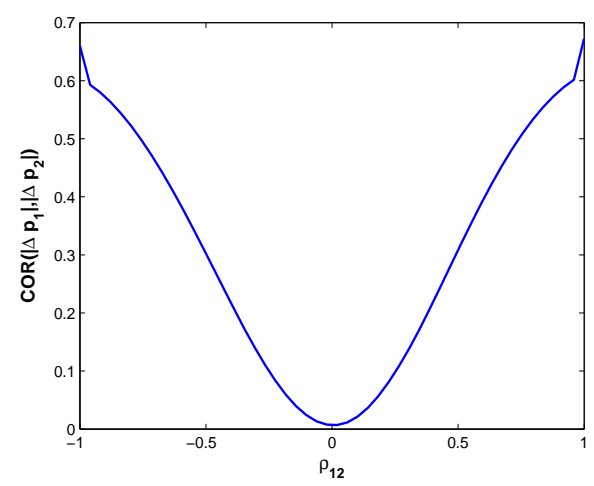

(c) The volatility correlation

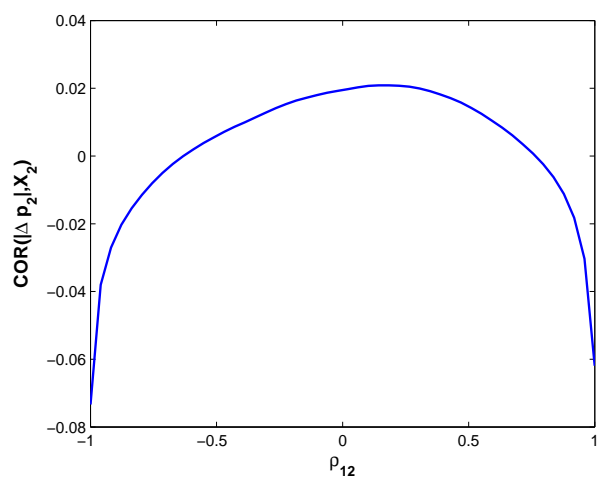

(b) Correlation between trading volume and volatility for asset two

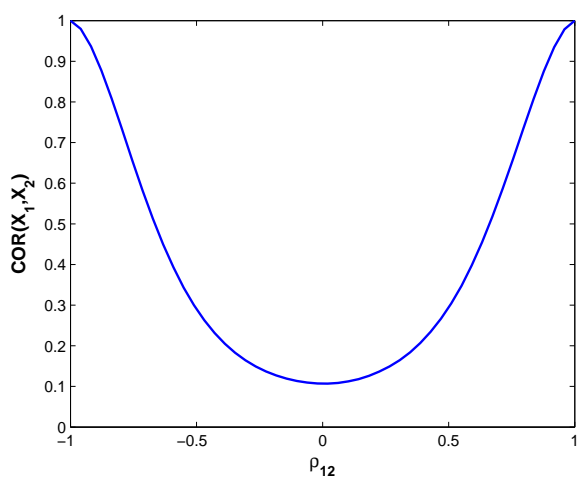

(d) The trading volume correlation

FiguRE 4.8. The correlation between trading volumes and volatilities for asset one (a) and asset two (b) and the correlations of price volatilities (c) and trading volumes (d) of the two risky assets. The results are based on 100 simulations with $\eta=1.5$.

trading volumes of the two assets are high when both assets are highly correlated. The result is very intuitive; when the payoffs are less correlated in agents' beliefs, both price volatility and trading volume of the two assets are also less correlated. In summary, the persistence in price volatility and trading volume and the autocorrelation patterns in volatility and trading volume illustrated by the model are closely related to the characteristics of financial markets. 


\section{Conclusion}

This paper extends the single-period equilibrium CAPM of Chiarella et al (2011) to a dynamic equilibrium evolutionary CAPM to incorporate the adaptively switching behaviour of heterogeneous agents. By analyzing the stability of the underlying deterministic model, we show that the evolutionary CAPM is capable of characterizing the spill-over effects, the persistence in price volatility and trading volume, and realistic correlations between price volatility and trading volume. Also, the stochastic nature of time-varying betas implied by the equilibrium model may not be consistent with the rolling window estimate of betas used in the empirical literature. The model provides further explanatory power of the recently developed HAMs.

In this paper, the numerical analysis is focused on the case of two risky assets, though the stability analysis is conducted for any number of risky assets. It would be interesting to see how an increase in the number of risky assets could have different effects. We expect the main results obtained in this paper to hold. The statistical analysis is mainly based on some Monte Carlo simulations and a systematical study of the empirical relevance using econometric methods would be interesting. We leave these issues to the future research.

\section{Appendix A. Proofs}

To provide some insights into the proof of the model with many risky assets, we first start with the case of one risky asset.

A.1. Proof of Proposition 3.2. In order to prove the local stability properties of the deterministic model (3.1), we start from the simplified one-risky-asset case (3.3). We omit the index $j$ of the unique risky asset for simplicity.

Note that $p_{t}$ depends only on $p_{t-1}, u_{t-1}, V_{t-1}$, and on $n_{f, t}$. The same holds for the state variables $u_{t}$ and $V_{t}$. The differential of the fitness functions at time $t, v_{\Delta, t}$, depends on $p_{t-2}, u_{t-2}, V_{t-2}$ and $p_{t-1}$ through the demand functions $z_{f, t-1}$ and $z_{c, t-1}$, on $n_{f, t-1}$ through $\theta_{a, t-1}$, as well as on $p_{t}, p_{t-1}, p_{t-2}$ directly. Formally, suitable changes of variables allow us to express the dynamical system (3.3) as an 8-dimensional map, by which the state of the system at time $t$ is expressed as a 
function of the state of the system at time $t-1$. We set

$$
q_{f, t}:=n_{f, t+1}=\left(1+\exp \left(-\eta v_{\Delta, t}\right)\right)^{-1}, \quad p_{t}^{L}:=p_{t-1}, \quad u_{t}^{L}:=u_{t-1}, \quad V_{t}^{L}:=V_{t-1}
$$

so that we can write the map driving dynamical system (3.3) as

$$
\begin{aligned}
p_{t} & =F\left(p_{t-1}, u_{t-1}, V_{t-1}, q_{f, t-1}\right) \\
u_{t} & =\delta u_{t-1}+(1-\delta) F\left(p_{t-1}, u_{t-1}, V_{t-1}, q_{f, t-1}\right), \\
V_{t} & =\delta V_{t-1}+\delta(1-\delta)\left[F\left(p_{t-1}, u_{t-1}, V_{t-1}, q_{f, t-1}\right)-u_{t-1}\right]^{2}, \\
q_{f, t} & =\left\{1+\exp \left[-\eta Q\left(p_{t-1}, u_{t-1}, V_{t-1}, q_{f, t-1}, p_{t-1}^{L}, u_{t-1}^{L}, V_{t-1}^{L}, q_{f, t-1}^{L}\right)\right]\right\}^{-1} .
\end{aligned}
$$

In particular, the function $Q$ in the fourth equation above corresponds to $v_{\Delta, t}$, and has the structure

$$
Q=v_{\Delta, t}=\hat{\zeta}_{f, t-1} \hat{\pi}_{f, t}-\hat{\zeta}_{c, t-1} \hat{\pi}_{c, t}-C_{\Delta}
$$

where, for $h \in\{f, c\}$,

$$
\hat{\zeta}_{h, t-1}:=z_{h, t-1}-\frac{\theta_{a, t-1}}{\theta_{h}} s, \quad \hat{\pi}_{h, t}:=p_{t}+\bar{d}-R_{f} p_{t-1}-\frac{\theta_{h}}{2} \sigma_{h, t-1}^{2}\left(z_{h, t-1}+\frac{\theta_{a, t-1}}{\theta_{h}} s\right)
$$

and $\sigma_{h, t-1}^{2}=\sigma^{2}$ for $h=f, \sigma_{h, t-1}^{2}=\sigma^{2}+\lambda V_{t-2}$ for $h=c$. One can check that both $\hat{\zeta}_{h, t-1}$ and $\hat{\pi}_{h, t}$ vanish at the fundamental steady state. It follows that all the partial derivatives of $Q$ with respect to any of the state variables also vanish at the steady state, and the same holds for the derivatives of $q_{f, t}$. Also, all the partial derivatives of $V_{t}$ (except $\partial V_{t} / \partial V_{t-1}$ ) are zero at the steady state due to the higher-order term $\left(p_{t}-u_{t-1}\right)^{2}$ and the fact that $p=u$ at the steady state. By ordering the variables as $p, u, V, q_{f}, p^{L}, u^{L}, V^{L}, q_{f}^{L}$, the Jacobian matrix evaluated at the fundamental steady state has the left block triangular structure

$$
\mathbf{J}=\left(\begin{array}{cc}
\mathbf{A} & \mathbf{0} \\
\mathbf{I} & \mathbf{0}
\end{array}\right)
$$

where $\mathbf{0}$ and $\mathbf{I}$ are the 4-dimensional null and identity matrices, respectively, and

$$
\mathbf{A}=\left(\begin{array}{cccc}
\frac{\partial F}{\partial p} & \frac{\partial F}{\partial u} & \frac{\partial F}{\partial V} & \frac{\partial F}{\partial q_{f}} \\
(1-\delta) \frac{\partial F}{\partial p} & \delta+(1-\delta) \frac{\partial F}{\partial u} & (1-\delta) \frac{\partial F}{\partial V} & (1-\delta) \frac{\partial F}{\partial q_{f}} \\
0 & 0 & \delta & 0 \\
0 & 0 & 0 & 0
\end{array}\right)
$$


It follows that the characteristic equation for $\mathbf{J}$ is given by

$$
\chi^{5}(\chi-\delta)\left(\chi^{2}+m_{1} \chi+m_{2}\right)=0
$$

where 16

$$
m_{1}=\frac{\alpha+\delta \gamma}{R_{f}\left(1+\theta_{0} e^{\eta C_{\Delta}}\right)}-\frac{\delta \gamma+1}{R_{f}}-\delta, \quad m_{2}=\delta\left[\frac{1+\gamma}{R_{f}}-\frac{\alpha+\gamma}{R_{f}\left(1+\theta_{0} e^{\eta C_{\Delta}}\right)}\right] .
$$

As $0<\delta<1$, it follows that stability depends only on the roots of the 2nd-degree polynomial $\chi^{2}+m_{1} \chi+m_{2}$. The latter represents the characteristic polynomial of the two-dimensional upper-left block of matrix A (that we denote as B). A wellknown necessary and sufficient condition for both characteristic roots of $\mathbf{B}$, say $\chi_{1}$ and $\chi_{2}$, to have modulus smaller than one (implying that the steady state is locally asymptotically stable in our case) is the set of inequalities,

$$
1+m_{1}+m_{2}>0, \quad 1-m_{1}+m_{2}>0, \quad m_{2}<1
$$

The first and second inequalities of (A.2) always hold for any $\eta \geq 0$. The third condition is equivalent to

$$
\delta(1+\gamma)-R_{f}<\frac{\delta(\alpha+\gamma)}{1+\theta_{0} e^{\eta C_{\Delta}}}
$$

If $R_{f} \geq \delta(1+\gamma)$, then condition (A.3) always holds for any $\eta \geq 0$. If $R_{f}<\delta(1+\gamma)$, then (A.3) holds when $\eta<\widehat{\eta}:=\frac{1}{C_{\Delta}} \ln \frac{R_{f}-\delta(1-\alpha)}{\theta_{0}\left[\delta(1+\gamma)-R_{f}\right]}$. If $C_{\Delta}=0$, then Eq. (A.3) holds when $\frac{R_{f}-\delta(1-\alpha)}{\theta_{0}\left[\delta(1+\gamma)-R_{f}\right]}>1$, which is equivalent to $\theta_{0} \gamma<\alpha+\left(1+\theta_{0}\right)\left(\frac{R_{f}}{\delta}-1\right)$. This proves Proposition 3.2.

A.2. Proof of Proposition 3.1. Consider the general case (3.1) of $N$ risky assets. The structure of the map is the same as in the simplified one-risky-asset case, except that the variables $\mathbf{p}_{t}, \mathbf{u}_{t}, \mathbf{p}_{t}^{L}, \mathbf{u}_{t}^{L}$ have dimension $N$, whereas $\mathbf{V}_{t}$ and $\mathbf{V}_{t}^{L}$ have dimension $M:=N(N+1) / 2$ (e.g. $M=3$ for the two-asset case). Again, $\mathbf{p}=\mathbf{u}$ at the steady state, and the derivatives of each component of $\mathbf{V}_{t}$ in system (3.1) with respect to any of the state variables (with the exception of $\mathbf{V}_{t-1}$ ) vanish

\footnotetext{
${ }^{16}$ See later for the $N$-asset case with the computational details regarding $\frac{\partial F}{\partial p}$ and $\frac{\partial F}{\partial u}$.
} 
at the steady state. Turning to the derivatives of $q_{f, t}:=n_{f, t+1}$, note that function $Q$ has the structure

$$
Q=v_{\Delta, t}=\hat{\boldsymbol{\zeta}}_{f, t-1}^{\top} \hat{\pi}_{f, t}-\hat{\boldsymbol{\zeta}}_{c, t-1}^{\top} \hat{\pi}_{c, t}-C_{\Delta}
$$

where, for $h=f, c$,

$\hat{\boldsymbol{\zeta}}_{h, t-1}:=\mathbf{z}_{h, t-1}-\frac{\theta_{a, t-1}}{\theta_{h}} \mathbf{s}, \quad \hat{\boldsymbol{\pi}}_{h, t}:=\mathbf{p}_{t}+\overline{\mathbf{d}}-R_{f} \mathbf{p}_{t-1}-\frac{\theta_{h}}{2} \boldsymbol{\Omega}_{h, t-1}\left(\mathbf{z}_{h, t-1}+\frac{\theta_{a, t-1}}{\theta_{h}} \mathbf{s}\right)$,

with $\boldsymbol{\Omega}_{h, t-1}=\boldsymbol{\Omega}_{0}$ for $h=f$ and $\boldsymbol{\Omega}_{h, t-1}=\boldsymbol{\Omega}_{0}+\lambda \mathbf{V}_{t-2}$ for $h=c$. Similar to the oneasset case, both $\hat{\boldsymbol{\zeta}}_{h, t-1}$ and $\hat{\boldsymbol{\pi}}_{h, t}$ vanish at the fundamental steady state, and the same holds for any of the partial derivatives of $q_{f, t}$. The Jacobian matrix of the system of dimension $N^{2}+5 N+2$ at the fundamental steady state is thus again characterized by the structure (A.1), where the variables are ordered as $\mathbf{p}, \mathbf{u}, \mathbf{V}, q_{f}, \mathbf{p}^{L}, \mathbf{u}^{L}, \mathbf{V}^{L}, q_{f}^{L}$. In particular, in this case, $\mathbf{0}$ and $\mathbf{I}$ represent the null matrix and the identity matrix of order $2 N+N(N+1) / 2+1$ (e.g. dimension 8 in the case of two assets) respectively, and 17

$$
\mathbf{A}=\left(\begin{array}{cccc}
D_{\mathbf{p}} \mathbf{F} & D_{\mathbf{u}} \mathbf{F} & D_{\mathbf{V}} \mathbf{F} & D_{q_{f}} \mathbf{F} \\
(1-\delta) D_{\mathbf{p}} \mathbf{F} & \delta \mathbf{I}+(1-\delta) D_{\mathbf{u}} \mathbf{F} & (1-\delta) D_{\mathbf{V}} \mathbf{F} & (1-\delta) D_{q_{f}} \mathbf{F} \\
\mathbf{0} & \mathbf{0} & \delta \mathbf{I} & \mathbf{0} \\
0 & 0 & 0 & 0
\end{array}\right)
$$

where $D_{\mathbf{x}} \mathbf{F}$ denotes the partial Jacobian matrix with respect to the variable $\mathbf{x}$. Again, what matters for stability are the eigenvalues of the upper left block (of dimension $2 N \times 2 N)$, given by

$$
\mathbf{B}=\left(\begin{array}{cc}
D_{\mathbf{p}} \mathbf{F} & D_{\mathbf{u}} \mathbf{F} \\
(1-\delta) D_{\mathbf{p}} \mathbf{F} & \delta \mathbf{I}+(1-\delta) D_{\mathbf{u}} \mathbf{F}
\end{array}\right) .
$$

Consider now the difference equation for the price vector

$$
\mathbf{p}_{t}=\mathbf{F}\left(\mathbf{p}_{t-1}, \mathbf{u}_{t-1}, \mathbf{V}_{t-1}, q_{f, t-1}\right)
$$

\footnotetext{
${ }^{17}$ The null matrices in the third row of $\mathbf{A}$ now have dimension $M \times N$ (first and second entry) and $M \times 1$ (fourth entry). The identity matrix in the third entry has dimension $M$. The identity matrix in the second row has dimension $N$.
} 
The partial Jacobian with respect to $\mathbf{p}$ is given by

$$
D_{\mathbf{p}} \mathbf{F}=\frac{\theta_{a, t}}{R_{f}} \boldsymbol{\Omega}_{a, t}\left[\frac{q_{f, t-1}}{\theta_{f}} \boldsymbol{\Omega}_{0}^{-1}(\mathbf{I}-\boldsymbol{\alpha})+\frac{1-q_{f, t-1}}{\theta_{c}}\left(\boldsymbol{\Omega}_{0}+\lambda \mathbf{V}_{t-1}\right)^{-1}(\mathbf{I}+\boldsymbol{\gamma})\right]
$$

where $\mathbf{I}$ is the $N$-dimensional identity matrix, $\boldsymbol{\alpha}:=\operatorname{diag}\left(\alpha_{1}, \alpha_{2}, \ldots, \alpha_{N}\right)$ and $\gamma:=\operatorname{diag}\left(\gamma_{1}, \gamma_{2}, \ldots, \gamma_{N}\right)$. At the steady state (where $\left.\boldsymbol{\Omega}_{a, t}=\boldsymbol{\Omega}_{0}\right)$ we obtain

$$
D_{\mathbf{p}} \mathbf{F}\left(\mathbf{p}^{*}, \mathbf{p}^{*}, \mathbf{0}, q_{f}^{*}\right)=\frac{\theta_{a}^{*}}{R_{f}}\left[\frac{n_{f}^{*}}{\theta_{f}}(\mathbf{I}-\boldsymbol{\alpha})+\frac{1-n_{f}^{*}}{\theta_{c}}(\mathbf{I}+\boldsymbol{\gamma})\right],
$$

where

$$
\frac{\theta_{a}^{*}}{\theta_{f}} n_{f}^{*}=\frac{1}{1+\theta_{0} e^{\eta C_{\Delta}}}, \quad \frac{\theta_{a}^{*}}{\theta_{c}}\left(1-n_{f}^{*}\right)=\frac{\theta_{0} e^{\eta C_{\Delta}}}{1+\theta_{0} e^{\eta C_{\Delta}}} .
$$

Note that $D_{\mathbf{p}} \mathbf{F}\left(\mathbf{p}^{*}, \mathbf{p}^{*}, \mathbf{0}, q_{f}^{*}\right)$ is a diagonal matrix. This implies that the fixed component $\Omega_{0}$ of variance/covariance beliefs, in particular the correlations, has no effect on the dynamics of the linearized system around the steady state. Similarly, one obtains for $D_{\mathbf{u}} \mathbf{F}$ the expression

$$
D_{\mathbf{u}} \mathbf{F}\left(\mathbf{p}^{*}, \mathbf{p}^{*}, \mathbf{0}, q_{f}^{*}\right)=-\frac{\theta_{a}^{*}}{R_{f}} \frac{1-n_{f}^{*}}{\theta_{c}} \gamma,
$$

which is also a diagonal matrix. Every submatrix of block $\mathbf{B}$ is therefore an $N$ dimensional diagonal matrix. It follows that the characteristic equation of $\mathbf{J}$ is given by

$$
\chi^{\frac{(N+1)(N+4)}{2}}(\chi-\delta)^{\frac{N(N+1)}{2}} \prod_{j=1}^{N}\left(\chi^{2}+m_{1, j} \chi+m_{2, j}\right)=0,
$$

where in particular the characteristic equation of $\mathbf{B}$ is represented by the product of the $N$ 2nd-degree polynomials, and the coefficients $m_{1, j}$ and $m_{2, j}$ have the same structure as those of the one-asset case, namely

$$
m_{1, j}=\frac{\alpha_{j}+\delta \gamma_{j}}{R_{f}\left(1+\theta_{0} e^{\eta C_{\Delta}}\right)}-\frac{\delta \gamma_{j}+1}{R_{f}}-\delta, \quad m_{2, j}=\delta\left[\frac{1+\gamma_{j}}{R_{f}}-\frac{\alpha_{j}+\gamma_{j}}{R_{f}\left(1+\theta_{0} e^{\eta C_{\Delta}}\right)}\right] .
$$

Each of the above second-order polynomials is naturally associated with one of the risky assets. The steady state $\left(\mathbf{p}^{*}, \mathbf{p}^{*}, \mathbf{0}, q_{f}^{*}\right)$ is thus locally asymptotically stable if and only if, for all $j \in\{1,2, \ldots, n\}$,

$$
1+m_{1, j}+m_{2, j}>0, \quad 1-m_{1, j}+m_{2, j}>0, \quad m_{2, j}<1 .
$$

Similar to the one-asset case, the first two inequalities hold for any $\eta \geq 0$. The above set of inequalities is thus satisfied for any $\eta \geq 0$ if $R_{f} \geq \delta\left(1+\gamma_{j}\right)$. If $R_{f}<\delta\left(1+\gamma_{j}\right)$, it is satisfied only if $\eta<\widehat{\eta}_{j}:=\frac{1}{C_{\Delta}} \ln \frac{R_{f}-\delta\left(1-\alpha_{j}\right)}{\theta_{0}\left[\delta\left(1+\gamma_{j}\right)-R_{f}\right]}$ or, in the particular case $C_{\Delta}=0$, 
if $\theta_{0} \gamma_{j}<\alpha_{j}+\left(1+\theta_{0}\right)\left(\frac{R_{f}}{\delta}-1\right)$. Since stability requires that condition (A.4) holds for all $j \in\{1,2, \ldots, n\}$, the statement of Proposition 3.1 follows.

\section{REFERENCES}

Ang, A. and Chen, J. (2007), 'CAPM over the long run: 1926 to 2001', Journal of Empirical Finance 14, 1-40.

Banerjee, S. and Kremer, I. (2010), 'Disagreement and learning: dynamic patterns of trade', Journal of Finance 65, 1269-1302.

Bollerslev, T. (1986), 'Generalized autoregressive conditional heteroskedasticity', Journal of Econometrics 31, 307-327.

Bollerslev, T., Engle, R. and Wooldridge, J. (1988), 'A capital asset pricing model with time varying covariances', Journal of Political Economy 96, 116-131.

Braun, P., Nelson, D. and Sunier, A. (1990), 'Good news, bad news, volatility and betas', Journal of Finance 50, 1575-1603.

Brock, W. and Hommes, C. (1997), 'A rational route to randomness', Econometrica 65, 1059-1095.

Brock, W. and Hommes, C. (1998), 'Heterogeneous beliefs and routes to chaos in a simple asset pricing model', Journal of Economic Dynamics and Control 22, 1235-1274.

Campbell, J. and Vuolteenaho, T. (2004), 'Bad beta, good beta', American Economic Review 94(5), 1249-1275.

Chen, S.-H. and Huang, Y. (2008), 'Risk preference, forecasting accuracy and survival dynamics: simulations based on a multi-asset agent-based artifical stock market', Journal of Economic Behavior and Organization 67, 702-717.

Chiarella, C., Dieci, R. and Gardini, L. (2005), 'The dynamic interaction of speculation and diversification', Applied Mathematical Finance 12(1), 17-52.

Chiarella, C., Dieci, R. and He, X. (2009), Heterogeneity, Market Mechanisms and Asset Price Dynamics, Elsevier, pp. 277-344. in Handbook of Financial Markets: Dynamics and Evolution, Eds. Hens, T. and K.R. Schenk-Hoppé.

Chiarella, C., Dieci, R. and He, X. (2010), A framework for CAPM with heterogeneous beliefs, Springer, pp. 353-369. in Nonlinear Dynamics in Economics, Finance and Social Sciences: Essays in Honour of John Barkley Rosser Jr., Eds. Bischi, G.-I., C. Chiarella and L. Gardini.

Chiarella, C., Dieci, R. and He, X. (2011), 'Do heterogeneous beliefs diversify market risk?', European Journal of Finance 17(3), 241-258.

Chiarella, C., Dieci, R. and He, X. (2012), 'Time-varying beta: A boundedly rational equilibrium approach', Journal of Evolutionary Economics . to appear (DOI 10.1007/s00191-011-0233-5).

Chiarella, C., He, X. and Zheng, M. (2011), 'An analysis of the effect of noise in a heterogeneous agent financial market model', Journal of Economic Dynamics and Control 35. 148-162. 
Dieci, R. and Westerhoff, F. (2010a), 'Heterogeneous speculators, endogenous fluctuations and interacting markets: A model of stock prices and exchange rates', Journal of Economic Dynamics and Control 34, 743-764.

Dieci, R. and Westerhoff, F. (2010b), On the inherent instability of international financial markets: natural nonlinear interactions between stock and foreign exchange markets, BERG (Bamberg Economic Research Group) on Government and Growth Working Paper Series, no. 79, University of Bamberg.

Dybvig, P. and Ross, S. (1985), 'Differential information and performance measurement using a security market line', Journal of Finance 40, 383-400.

Engle, R. (1982), 'Autoregressive conditional heteroscedasticity with estimates of the variance of UK inflation', Econometrica 50, 987-1008.

Evstigneev, I., Hens, T. and Schenk-Hoppé, K.R. (2009), Evolutionary Finance, Elsevier, pp. 507566. in Handbook of Financial Markets: Dynamics and Evolution, Eds. Hens, T. and K.R. Schenk-Hoppé.

Fama, E. and French, K. (2006), 'The value premium and the CAPM', Journal of Finance 61(5), 2163-2185.

Hamilton, J. (1989), 'A new approach to the economic analysis of nonstationary time series and the business cycle', Econometrica 57(2), 357-384.

Hamilton, J. (1990), 'Analysis of time series subject to changes in regime', Journal of Econometrics 45, 39-70.

Hansen, L. and Richard, S. (1987), 'The role of conditioning information in deducing testable restrictions implied by dynamic asset pricing models', Econometrica 55, 587-613.

He, X. and Li, Y. (2007), 'Power law behaviour, heterogeneity, and trend chasing', Journal of Economic Dynamics and Control 31, 3396-3426.

Hens, T. and Schenk-Hoppé, K. R., eds (2009), Handbook of Financial Markets: Dynamics and Evolution, Handbooks in Finance, Elsevier.

Hommes, C. (2001), 'Financial markets as nonlinear adaptive evolutionary systems', Quantitative Finance 1, 149-167.

Hommes, C. (2006), Heterogeneous Agent Models in Economics and Finance, Vol. 2 of Handbook of Computational Economics, North-Holland, pp. 1109-1186. in Agent-based Computational Economics, Eds. Tesfatsion, L. and K.L. Judd.

Hommes, C. and Wagener, F. (2009), Complex Evolutionary Systems in Behavioral Finance, Chapter 4 in 'Handbooks in Finance', North-Holland, pp. 217-276. in Handboof of Financial Markets: Dynamics and Evolution, Eds. Hens, T. and K.R. Schenk-Hoppé.

Jagannathan, R. and Wang, Z. (1996), 'The conditional CAPM and cross-section of expected returns', Journal of Finance 51, 3-53. 
LeBaron, B. (2006), Agent-based Computational Finance, Vol. 2 of Handbook of Computational Economics, North-Holland, pp. 1187-1233. in Agent-based Computational Economics, Eds. Tesfatsion, L. and K.L. Judd.

Lewellen, J. and Nagel, S. (2006), 'The conditional CAPM does not explain asset-pricing anomalies', Journal of Financial Economics 82(3), 289-314.

Lintner, J. (1965), 'The valuation of risk assets and the selection of risky investments in stock portfolios and capital budgets', Review of Economic Studies 47, 13-37.

Lux, T. (2009), Stochastic Behavioural Asset Pricing and Stylized Facts, Elsevier, pp. 161-215. in Handbook of Financial Markets: Dynamics and Evolution, Eds. Hens, T. and K.R. SchenkHoppé.

Marsili, M., Raffaelli, G. and Ponsot, B. (2009), 'Dynamic instability in generic model of multiassets markets', Journal of Economic Dynamics and Control 33, 1170-1181.

Mossin, J. (1966), 'Equilibrium in a capital asset market', Econometrica 35, 768-783.

Sharpe, W. (1964), 'Capital asset prices: A theory of market equilibrium under conditions of risk', Journal of Finance 19, 425-442.

Westerhoff, F. (2004), 'Multiasset market dynamics', Macroeconomic Dynamics 8, 591-616.

Westerhoff, F. and Dieci, R. (2006), 'The effectiveness of Keynes-Tobin transaction taxes when heterogeneous agents can trade in different markets: A behavioral finance approach', Journal of Economic Dynamics and Control 30, 293-322.

Zhu, M., Wang, D. and Guo, M. (2011), 'Stochastic equilibria of an asset pricing model with heterogeneous beliefs and random dividends', Journal of Economic Dynamics and Control 35, 131-147. 